\title{
Influence of Different Types of Small-Size Defects on Propagation of Macro-cracks in Brittle Materials
}

\section{Olena Karpenko $^{1} \cdot$ Selda Oterkus ${ }^{1} \cdot$ Erkan Oterkus $^{1}$}

Received: 23 December 2019 / Accepted: 8 April 2020 / Published online: 30 April 2020

(C) The Author(s) 2020

\begin{abstract}
The presence of defects in the structure requires noticeable attention and understanding of fracture mechanisms in brittle materials has to be established. Defects in the form of holes, macro- and micro-cracks are the main interest of this paper. This work investigates the dual role of holes and micro-crack arrays on toughening and degradation mechanisms in concrete structures. An ordinary state-based peridynamics (PD) model is utilized to analyse the fracture problem at the micro-level. The application of PD shows its advantage in crack-hole, macro- and micro-crack interaction problems since PD can accurately predict the contribution of defects on structural behaviour. The study of the three-point bending problem with five types of holes existing in the structure showed the crack arrest phenomena at the hole boundary and the "attraction" of the crack to propagate towards the hole. For the study of the macro- and micro-cracks interaction problem, various cases of the micro-crack distribution and inclination angles are considered and validated with analytical studies. The PD quasi-static simulations show good agreement with analytical solutions. Moreover, PD dynamic solutions show the capability of PD to capture complex crack propagation paths. It is observed that the presence of micro-cracks and holes ahead of the main crack can suppress its further propagation as well as have an influence on the crack propagation direction. The numerical results demonstrate the efficiency of the PD modelling of multiple crack interaction problems.
\end{abstract}

Keywords Peridynamics $\cdot$ Brittle fracture $\cdot$ Defects $\cdot$ Micro-cracks $\cdot$ Nonlocal

\section{Introduction}

The failure of engineering structures has always been a topic of engineering practice. One of the common types of mechanical failure is a brittle fracture [1-4]. Mechanical failure and

Selda Oterkus

selda.oterkus@strath.ac.uk

1 Department of Naval Architecture, Ocean and Marine Engineering, University of Strathclyde, 100 Montrose Street, Glasgow, UK 
defects in brittle materials can lead to weakening and the sudden collapse of the structure. Considering that the brittle materials, such as concrete, ceramics and rocks, are widely used in various industries, it is important to investigate their structural behaviour. Brittle materials can consist of randomly distributed small-size defects such as voids, micro-cracks and holes [5]. These defects can have a significant influence on the propagation behaviour of macro-cracks. There have been various experimental studies in the literature which investigate the interaction between macro-cracks and small-size defects [6-9]. Different studies suggest that the existence of micro-cracks can lead to "micro-crack toughening phenomena" [10-12]. The presence of micro-cracks can act as the suppressors of the macro-crack propagation and contribute to the increase of the fracture zone [13]. Moreover, depending on the distribution, distances, sizes and inclination angles of micro-cracks, micro-cracks interacting with a macro-crack can lead to stress shielding effects with the decrease of stress intensity factor (SIF) $[5,11,14]$. Multiple analytical studies were performed for problems with a single micro-crack ahead of the main crack [15], the interaction between the main crack with multiple micro-crack arrays [11] and the surrounding micro-cracks [16]. The applied methods analyse the stress fields ahead of the crack tip, characterised by $K_{0} S$ for the models with a single macro-crack and $K_{I}$ SIF for various distributions of micro-cracks, and describe the macro- and micro-crack interaction by using the parameter $K_{I} / K_{0}$. The parameter $K_{I} / K_{0}$ is helpful to identify if micro-cracks enhance or suppress the macro crack propagation and is very useful for understanding the complex structural behaviour with defects. Besides, when the plate consists of both macro- and micro-cracks, micro-cracks can have influence on the main crack propagation behaviour where the main crack follows the orientation of micro-cracks. The classical continuum mechanics based approaches were used to solve the complex problems of crack interactions, but assumptions were made to simplify the problem. For example, analytical calculations include only the interaction of the main crack with micro-cracks, but the mutual interaction between the micro-cracks is neglected $[17,18]$.

On the other hand, the studies of the crack-hole interaction resulted in the momentary crack arrest phenomena $[19,20]$. These studies showed that the fracture in the specimen was momentarily interrupted for several microseconds, then reinitiated usually with the increased crack propagation speed. Such behaviour is noticed in the structure when the hole defects are present ahead of the propagating crack. The location and dimensions of the holes, loading rates have an effect on whether propagation of the crack can be stopped or it can propagate through the holes. It was investigated that when the hole lying eccentrically at some distance from the axis of the main crack, the propagating crack deflects towards the hole and then recovers its initial trajectory. As concluded by authors in [19], the asymmetry of the stress field in the area of the hole resulted in the curvature of the propagating crack with the crack arrest phenomena. The change of the parameter $K_{I} / K_{0}$ was also discussed in [21] by mentioning that the values of the SIF are increasing during the crack arrest period. An integral equation approach was proposed in [22] to investigate the hole-crack arrangements and stated that the distribution of holes can lead to the toughening phenomena in the structure as well. Therefore, the sizes and distribution of the holes have a major impact on stress reduction or amplification.

The problem of the presence of defects in the structure has attracted significant interest in the literature. Although experimental fracture analyses are very valuable, experimental tests can be costly and the laboratory experiments have to be carefully controlled to obtain accurate results. On the other hand, numerical tools are an effective alternative to experiments for studying fracture behaviour and mechanisms. Finite element method (FEM) was used to predict brittle fracture and simulate crack propagation and branching. FEM is based on 
classical continuum mechanics equations which are in the form of partial differential equations. To simulate crack propagation, the tracking of the crack surface is required. Moreover, it is necessary to determine the crack propagation direction and evaluation of the stress intensity factors for the crack $[23,24]$. Moreover, FEM is mesh-dependent and requires to remesh the model after each propagation increment to solve the problem of the crack growth. To overcome these difficulties, the extended finite element method (XFEM) was developed to solve problems containing discontinuities like cracks $[25,26]$, where the crack is modelled independently of mesh. XFEM has been successfully applied to a number of macro- and micro-crack interaction problems [27-29], but still requires to formulate the position and the shape of the crack tip.

As an alternative, a new numerical method for fracture analysis, peridynamics (PD), was developed by Silling in [30] which overcomes the limitations of classical continuum mechanics. PD models use integro-differential equations instead of partial differential equations as in classical continuum mechanics. PD formulation allows to deal with complex crack interaction problems where multiple cracks of arbitrary shapes can be introduced in the structure, and tracking of the crack tip location and crack behaviour is not necessary. For this reason, original "bond-based" PD has been successfully applied to brittle fracture problems to predict crack nucleation and propagation [31-34] as well as the main crack interacting with micro-cracks $[35,36]$. However, the wide application of the "bond-based" PD [37] model has limitations in the specification of material properties since Poisson's ratio is constrained to $1 / 3$ in the $2 \mathrm{D}$ models and $1 / 4$ in the $3 \mathrm{D}$ models. To eliminate this limitation, ordinary state-based PD was proposed by Silling et al. [38]. The improved ordinary state-based PD model provides more realistic results when Poisson's ratio is different than $1 / 3$ for 2D models and $1 / 4$ for $3 \mathrm{D}$ models.

In this study, ordinary state-based PD model is used to investigate the influence of smallsize defects on the propagation of macro-cracks in brittle materials. In "Section 2", ordinary state-based PD is briefly introduced. In "Section 3", the PD simulations are presented for a crack-hole interaction problem under three-point bending loading. First of all, the PD model is verified with finite element analysis (FEA) and validated with experimental results [39], where five types of hole defects are allocated in the plate. Secondly, the validated PD model is applied for the prediction of mechanical failure in a concrete structure. In "Section 4", PD simulations are performed for the main crack interacting with the micro-cracks problem. Five different cases of micro-crack presence under quasi-static boundary conditions (BCs) are presented and the determined critical loads are compared against analytical solutions [17]. Considering the importance of the micro-cracks on main crack propagation behaviour, the influence of the parameter $K_{I} / K_{0}$ on critical load is analysed to understand the "micro-crack toughening phenomena". Furthermore, the PD models with varying micro-crack distribution and orientation are analysed under dynamic BCs. The obtained PD numerical results of crack propagation patterns and crack propagation speeds are presented. Finally, the conclusions are given in "Section 5".

\section{Peridynamic Formulation}

The ordinary state-based PD introduced by Silling [40] is a reformulation of the fundamental equations of continuum mechanics equations which are particularly suitable to solve problems including discontinuities. PD uses integro-differential equations instead of partial differential 
equations as in classical continuum mechanics. This widens the possibility of solving fracture mechanics problems, including cracks initiation and propagation [41].

The peridynamic equation of motion can be written in the form of the integro-differential equation as follows:

$$
\rho \ddot{u}(x, t)=\int_{H}\left(t\left(u^{\prime}-u, x^{\prime}-x, t\right)-t\left(u-u^{\prime}, x-x^{\prime}, t\right)\right) d V^{\prime}+b(x, t)
$$

which can be discretized as follows:

$$
\rho_{(i)} \ddot{u}_{(i)}=\sum_{j=1}^{N}\left(t_{(i)(j)}\left(u_{(j)}-u_{(i)}, x_{(j)}-x_{(i)}, t\right)-t_{(j)(i)}\left(u_{(i)}-u_{(j)}, x_{(i)}-x_{(j)}, t\right)\right) V_{(j)}+b_{(i)}
$$

from which the acceleration $\ddot{u}_{(i)}$ of the material point $i$ at time $t$ can be obtained. Each material point $i$ interacts with other material points $j$ within its horizon $H_{x_{(i)}}$ with a total number $N$ of the family members for the point $i$, as shown in Fig. 1. The coordinates of a material point are represented as $x$ with the incremental volume $V . u, b$ and $\rho$ denote displacement vector field, body load and mass density of the material point, respectively. Conducting the simulations for 2-dimensional problems, the horizontal and vertical displacements are addressed as $u$ and $v$, respectively.

In the ordinary state-based PD, interacting material points can exert forces on each other with unequal magnitudes [42] and for the interacting material points $i$ and $j$, peridynamic force densities can be defined as follows:

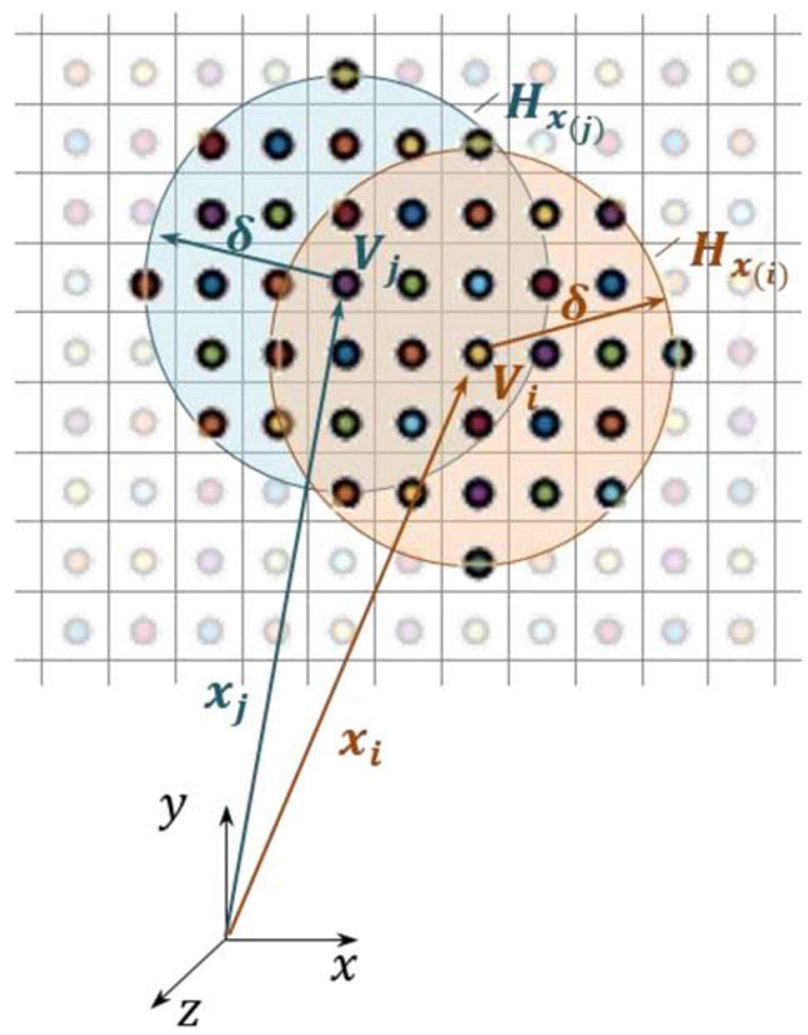

Fig. 1 Peridynamic material points and interaction of material points $i$ and $j$ 


$$
\begin{gathered}
t_{(i)(j)}=2 \delta\left\{d \frac{\Lambda_{(i)(j)}}{\left|x_{(j)}-x_{(i)}\right|} a \theta_{(i)}+2 \delta b s_{(i)(j)}\right\} \times \frac{y_{(j)}-y_{(i)}}{\left|y_{(j)}-y_{(i)}\right|} \\
t_{(j)(i)}=-2 \delta\left\{d \frac{\Lambda_{(i)(j)}}{\left|x_{(j)}-x_{(i)}\right|} a \theta_{(j)}+2 \delta b s_{(i)(j)}\right\} \times \frac{y_{(j)}-y_{(i)}}{\left|y_{(j)}-y_{(i)}\right|}
\end{gathered}
$$

where $\delta$ is the horizon size; $a, b, d$ are the peridynamic parameters; $s$ is the stretch between material points; and $\theta$ is dilatation, which can be expressed as follows:

$$
\theta_{(i)}=d \delta \sum_{j=1}^{N} \Lambda_{(i)(j)} S_{(i)(j)} V_{(j)}
$$

and the parameter $\Lambda_{(i)(j)}$ is defined as follows:

$$
\Lambda_{(i)(j)}=\left(\frac{y_{(j)}-y_{(i)}}{\left|y_{(j)}-y_{(i)}\right|}\right) \cdot\left(\frac{x_{(j)}-x_{(i)}}{\left|x_{(j)}-x_{(i)}\right|}\right)
$$

Once a material point displaces to a new location as a result of deformation of the structure, its new location is specified as $y$ in the deformed configuration, as shown in Fig. 2.

Peridynamic parameters $a, b, d$ can be related to material constants of classical continuum mechanics by equating strain energy density of a material point inside a body subjected to isotropic expansion and simple shear loading conditions calculated from classical continuum mechanics and PD [42]. In this paper, a 2-dimensional model with

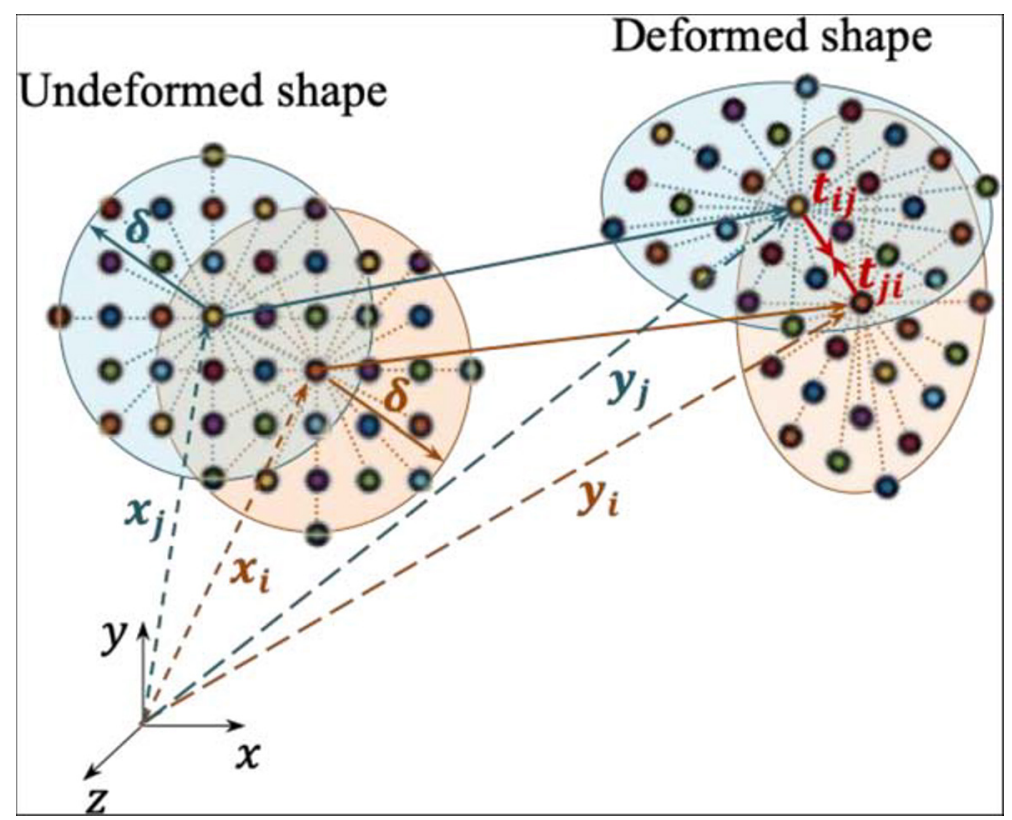

Fig. 2 Peridynamic material points $i$ and $j$ in the deformed configuration and the peridynamic forces between these material points 
plane stress conditions is used, where the plate is discretised with a single layer of material points in the thickness direction. Therefore, the PD parameters are expressed in terms of bulk modulus, $\kappa$; shear modulus, $\mu$; thickness, $h$; and horizon size, $\delta$, for a 2 dimensional problem as follows:

$$
a=\frac{1}{2}(\kappa-2 \mu), b=\frac{6 \mu}{\pi h \delta^{4}}, d=\frac{2}{\pi h \delta^{3}}
$$

The stretch between material points $i$ and $j$ can be expressed as follows:

$$
s_{(i)(j)}=\frac{\left(\left|y_{(j)}-y_{(i)}\right|-\left|x_{(j)}-x_{(i)}\right|\right)}{\left|x_{(j)}-x_{(i)}\right|}
$$

The failure parameter introduced by Silling and Bobaru [43] includes a history-dependent scalar-valued function $\mu$ to represent broken interactions (bonds) between material points

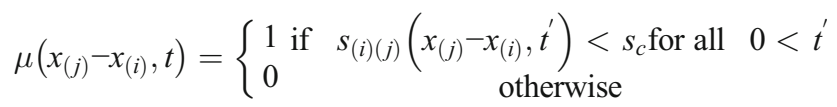

In Eq. (9), $s_{c}$ is the critical stretch [42] which can be expressed for a 2-dimensional problem as follows:

$$
s_{\mathrm{c}}=\sqrt{\frac{G_{c}}{\left(\frac{6}{\pi} \mu+\frac{16}{9 \pi^{2}}(\kappa-2 \mu)\right) \delta}}
$$

where $G_{c}$ is the critical energy release rate.

The local damage of the material point $i$, ranges from 0 to 1 , can be defined as follows:

$$
\varphi\left(x_{(i)}, t\right)=1-\frac{\sum_{i=1}^{N} \mu\left(x_{(j)}-x_{(i)}, t\right) V_{(j)}}{\sum_{i=1}^{N} V_{(j)}}
$$

\section{Influence of the Hole Defects on Crack Propagation in Three-Point Bending Test}

In this section, a three-point bending test is considered to analyse the crack propagation behaviour when hole defects are existing in the structure. Firstly, the PD model under static load is verified with FEA to set up the boundary conditions. Secondly, the solution is presented for crack propagation behaviour under dynamic loading with the presence of the defects. Five different types of holes are selected for the numerical analysis. The effect of the hole in the structure is investigated for a PMMA plate in order to verify the numerical PD model with the experimental tests [39]. Besides, the validated model is applied on a concrete plate for a crack behaviour analysis. 
A series of PD simulations are conducted to study the influence of Young's modulus, density, Poisson's ratio and fracture energy on crack behaviour in the presence of hole defects. Moreover, the influence of hole dimensions on crack propagation and crack arrest phenomena is investigated. Finally, the impact of different velocity boundary conditions (BC) on the crack path and crack propagation speed is analysed.

The explicit time integration scheme is used for all PD simulations. To achieve a stable solution, time step size $\Delta t$ has to be identified. For quasi-static problems ("Section 3.1"), the adaptive relaxation technique [41] is used to reach the steady-state solution and time step size $\Delta t=1$ is utilised as suggested in [41]. For dynamic problems with propagating crack in a system, the stability criterion on $\Delta t$ was introduced in [40] and used for the "Sections 3.2 and 3.3" of the presented work. Taking into account that stable time step size is dependent on horizon size, which is $\delta=3.015 \Delta x$, and effected by the mass density of the material, all dynamic computations in "Section 3" on PMMA are performed with $\Delta t=0.072 \mu$ s, while for concrete with $\Delta t=0.057 \mu \mathrm{s}$.

\subsection{Benchmark Problem}

A rectangular plate with a length of $L=0.2 \mathrm{~m}$, a width of $W=0.05 \mathrm{~m}$ and thickness of $h=$ $0.005 \mathrm{~m}$ in Fig. $3 \mathrm{a}$ is subjected to three-point bending loading. The supports are located $L_{s}=$ $0.14 \mathrm{~m}$ from each other. The pre-existing crack of $2 a=0.005 \mathrm{~m}$ is located at the bottom edge of the plate. The following material properties of the PMMA plate are specified: Young's modulus, $E=6.1 \mathrm{GPa}$ and Poisson's ratio, $\nu=0.31$.

The plate is subjected to the displacement constraints of $v(x, y=W, t)=5 \times 10^{-3} \mathrm{~m}$ and fully fixed supports at $y=0$. BCs are enforced in the following way (Fig. 3b.):

$$
\begin{gathered}
u_{f}\left(x_{f}, y_{f}, t+\Delta t\right)=0 \quad \text { at }-2 \Delta x \leq x \leq 2 \Delta x \quad \text { with } x_{f} \in R_{f(-2 \Delta x \leq x \leq 2 \Delta x)} \\
v_{f}\left(x_{f}, y_{f}, t+\Delta t\right)=-v(x, y, t+\Delta t) \text { at } \quad W \leq y \leq W+\delta \quad \text { with } y_{f} \in R_{f(W \leq y \leq W+\delta)} \\
u_{f_{s}}\left(x_{f_{s}}, y_{f_{s}}, t+\Delta t\right)=0 \\
v_{f_{s}}\left(x_{f_{s}}, y_{f_{s}}, t+\Delta t\right)=0
\end{gathered}
$$

Displacement boundary conditions are applied at the fictitious region $R_{f}$ located in the middle of the top edge of the plate. The PD model is discretised with 430 material points with uniform spacing between them $\Delta x=0.005 \mathrm{~m}$ and horizon size of $\delta=3.015 \Delta x$. A total time step of 2000 is sufficient to reach the quasi-static loading condition by means of adaptive dynamic relaxation method with a time step size of $\Delta t=1 \mathrm{~s}$ [41]. The supports are set up as a fully fixed displacement and velocity $\mathrm{BC}$ in a fictitious region $R_{f_{s}}$ at the bottom side of the plate. The width of the $R_{f_{s}}$ regions are equal to the horizon size of $\delta=3.015 \Delta x$. The peridynamic results are compared against FEA results obtained by using ANSYS, a commercial finite element 


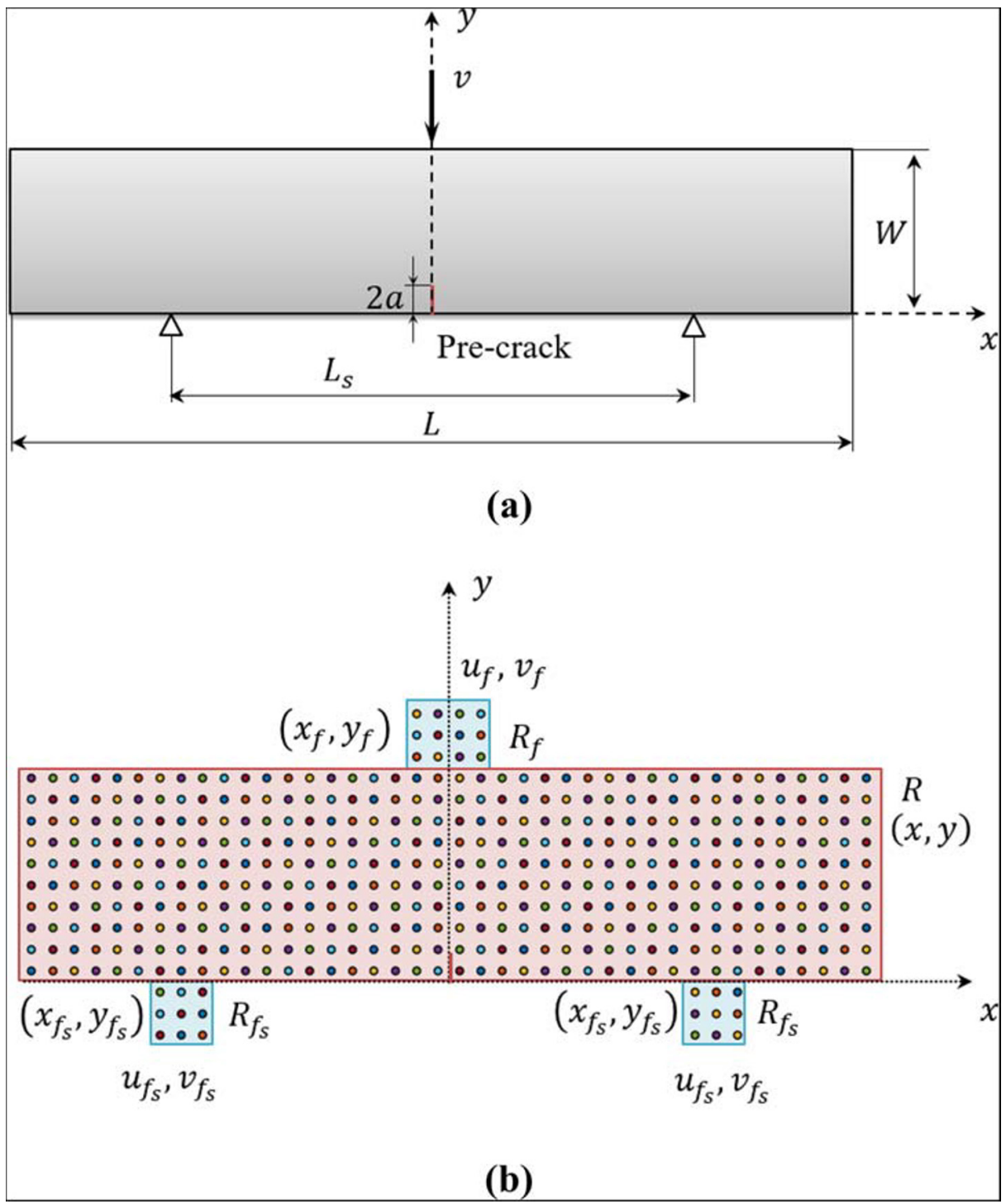

Fig. 3 a Pre-cracked rectangular PMMA plate under bending loading and $\mathbf{b}$ its discretisation

software. As shown in Fig. 4, the PD results are in good agreement with FEA results for the displacements along the centre lines of the plate.

\subsection{Hole Defects in PMMA}

PMMA plate considered in the previous case ("Section 3.1") is further investigated under dynamic loading conditions and including a pre-existing crack, as shown in Fig. 5 [39]. The pre-existing crack of $2 a=0.005 \mathrm{~m}$ is located at the bottom edge of the plate and five cases are selected with different values of the hole radius $R: R=0$, only pre-existing crack is allocated, $R=0.002 \mathrm{~m}, R=0.003 \mathrm{~m}, R=0.004 \mathrm{~m}, R=0.005 \mathrm{~m}$. The hole is located at a distance of $d_{x}=$ 


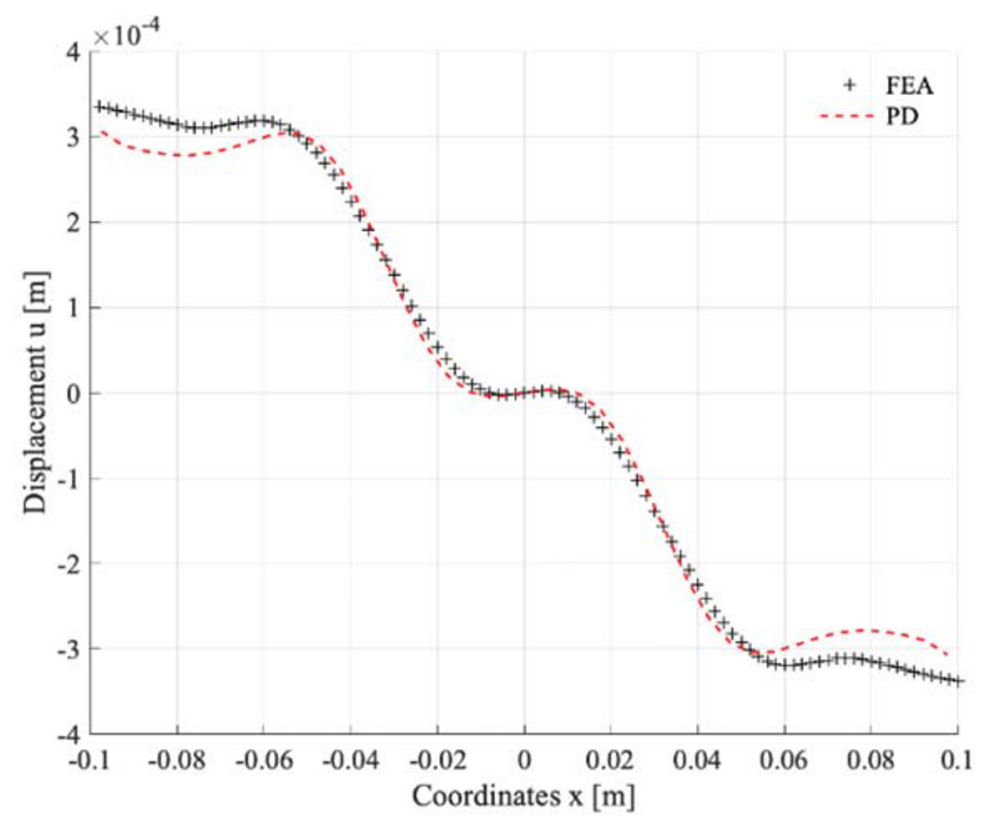

(a)

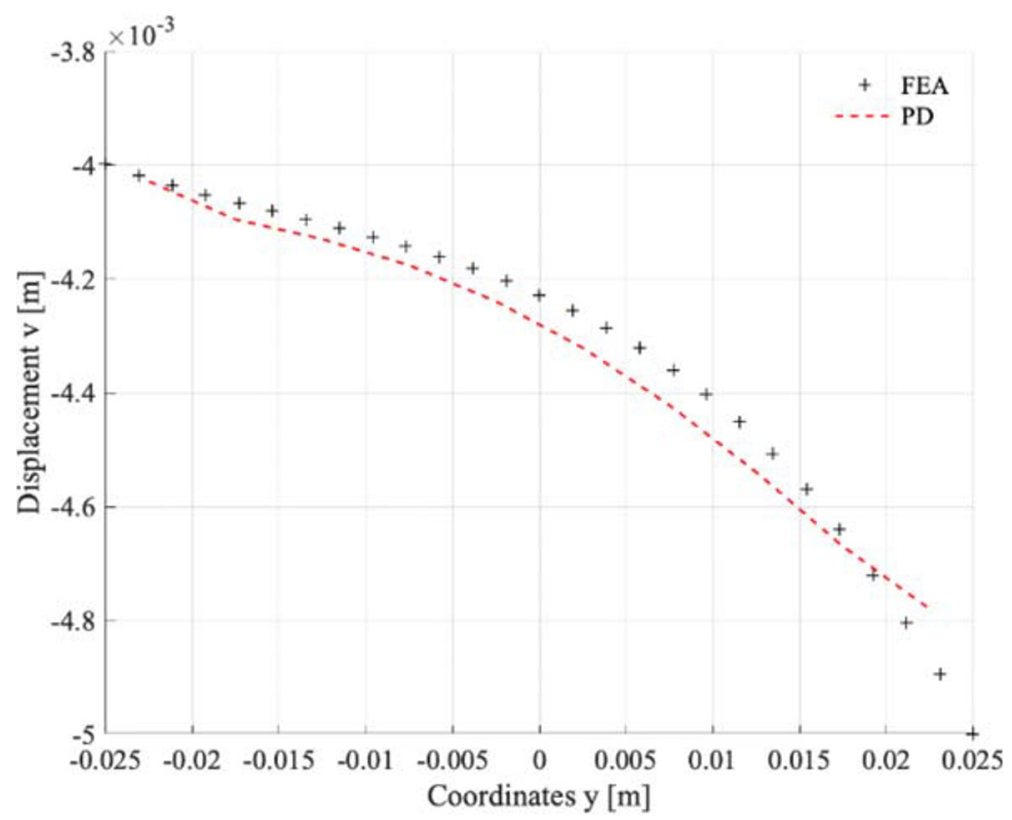

(b)

Fig. 4 Displacement variations along the centre lines $\mathbf{a} u(x, y=0)$ and $\mathbf{b} v(x=0, y)$ 


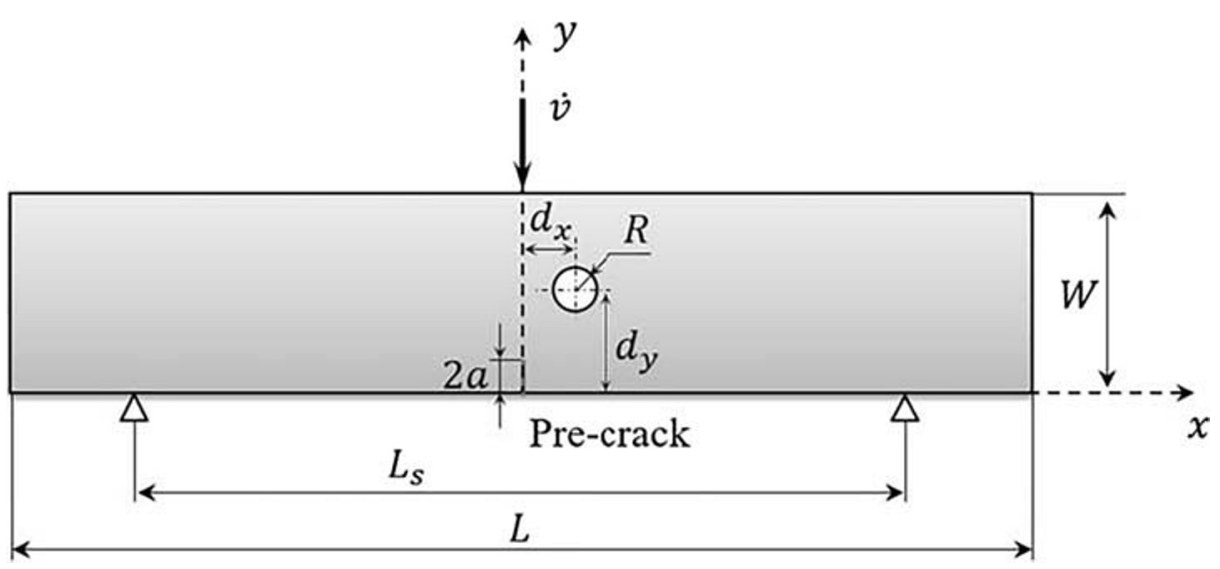

Fig. 5 Pre-notched rectangular PMMA plate with a hole defect under bending

$0.003+R \mathrm{~m}$ and $d_{y}=0.025 \mathrm{~m}$ from the origin of the main coordinate system located at the bottom of the crack. Velocity boundary conditions of (a) $\dot{v}=3 \mathrm{~m} / \mathrm{s}$ and (b) $\dot{v}=5 \mathrm{~m} / \mathrm{s}$ are applied at the fictitious region at the top edge of the plate (Fig. 3b) as following:

$$
\begin{aligned}
& u_{f}\left(x_{f}, y_{f}, t+\Delta t\right)=0 \quad \text { at }-2 \Delta x \leq x \leq 2 \Delta x \quad \text { with } x_{f} \in R_{f(-2 \Delta x \leq x \leq 2 \Delta x)} \\
& v_{f}\left(x_{f}, y_{f}, t+\Delta t\right)=-\dot{v}(x, y, t+\Delta t) \times \Delta t \quad \text { at } \quad W \leq y \leq W \\
&+\delta \quad \text { with } y_{f} \in R_{f(W \leq y \leq W+\delta)}
\end{aligned}
$$

The PD models are discretised with 160,000 material points with uniform spacing between them $\Delta x=0.00025 \mathrm{~m}$ and horizon size of $\delta=3.015 \Delta x$. Uniform time step size of $\Delta t=$ $0.072 \mu \mathrm{s}$ is used. The critical stretch is specified as $s_{c}=0.01072$ corresponding to an energy release rate of $G_{0}=220 \mathrm{~J} / \mathrm{m}^{2}$.

The crack propagation behaviour for different loading conditions from PD analysis is shown in Fig. 6a, b. When there is no hole defect in the structure, the crack propagates in a self-similar fashion. On the other hand, when hole defects are allocated in the structure, the crack path changes and deflects towards the holes. The same pattern of crack propagation is noticed during the experimental tests [39], shown in Fig. 6c. A higher deflection is observed for larger holes where the crack approaches the defect and after propagating close to the defect, it restores its original path. A curved crack trajectory can be noticed which is almost symmetrical with respect to the local axis of the hole. For both velocity $\mathrm{BC}$, the crack moves towards the hole and then it is momentarily arrested at the boundary of the hole without intersecting with the hole. It then moves further towards the location of the applied $\mathrm{BC}[19]$.

The crack propagation speed is evaluated and calculated as follows:

$$
V=\frac{x_{p}-x_{p-1}}{t_{p}-t_{p-1}}
$$



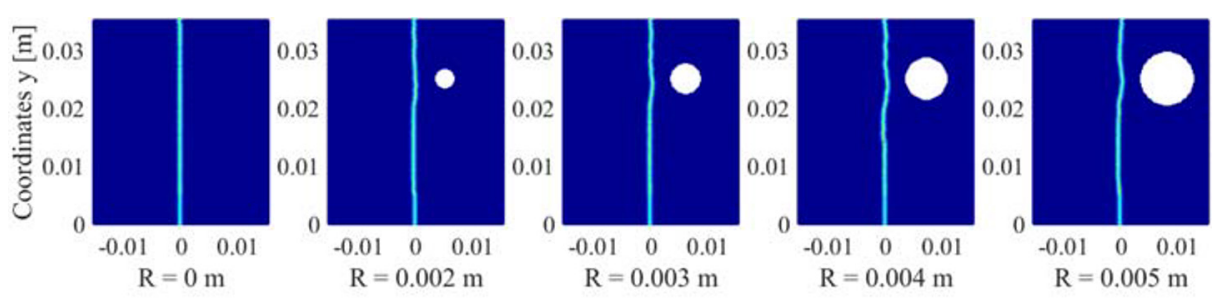

(a)
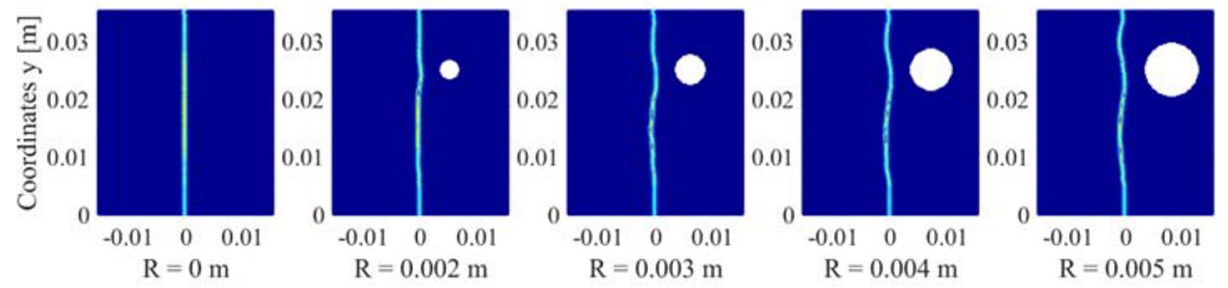

(b)
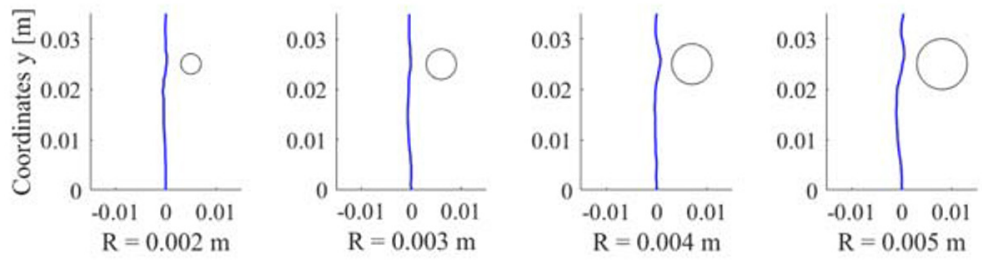

(c)

Fig. 6 PD Damage maps of the PMMA plate with different values of the hole radius $R$ and under velocity BC: a $\dot{v}=3 \mathrm{~m} / \mathrm{s}$ at time $t=360 \mu \mathrm{s}$ and $\mathbf{b} \dot{v}=5 \mathrm{~m} / \mathrm{s}$ at $t=230 \mu \mathrm{s} ; \mathbf{c}$ crack paths from the experimental study [39]

where $x_{p}$ and $x_{p-1}$ are the crack tip position at the current time $t_{p}$ and previous time $t_{p-1}$, respectively.

Figure 7a shows the variation of the crack propagation speed with increase in crack growth for velocity $\mathrm{BC}$ of $\dot{v}=3 \mathrm{~m} / \mathrm{s}$ when a hole with a radius of $R=0.005 \mathrm{~m}$ is inserted on the structure. It can be noticed that the crack propagation speed increases to the value of $V=$ $500 \mathrm{~m} / \mathrm{s}$ as the crack tip is at $x=0 \mathrm{~m}, y=\left(d_{y}-0.01\right) \mathrm{m}$ with the crack approaching the hole. As the crack propagates further and the crack trajectory approaches to the hole, the crack propagation speed gradually decreases up to $V=100 \mathrm{~m} / \mathrm{s}$. When the crack tip location is at $x=6 \times 10^{-4} \mathrm{~m}, y=d_{y}=0.023 \mathrm{~m}$ (the local axes of the hole is at $d_{x}=0.007 \mathrm{~m}, d_{y}=0.023 \mathrm{~m}$ ) with the crack is being arrested for around $10 \mu s$ (Fig. 7b) and then recovers its original trajectory with continually increasing speed. A similar variation of the crack propagation speed is noticed for the velocity $\mathrm{BC}$ of $\dot{v}=5 \mathrm{~m} / \mathrm{s}$ (Fig. 7a) where the crack propagation speed is higher and reaches $V=600 \mathrm{~m} / \mathrm{s}$. Furthermore, the crack is curving towards the hole with a gradually dropping speed to $V=250 \mathrm{~m} / \mathrm{s}$, and after a very small crack arresting period of $3 \mu \mathrm{s}$ (Fig. 7b), it leads to further recovery of the crack speed while propagates away from the hole. The phenomena of the crack arrest were noticed in the multiple tests $[19,20]$, where the crack propagation behaviour is very similar to the results achieved by PD simulations. It is not easy to detect the crack arrest phenomena as the crack arrest period is very short. Moreover, it can be noticed that the crack arrest is force dependent (Fig. 7b) with the increased delay of crack 

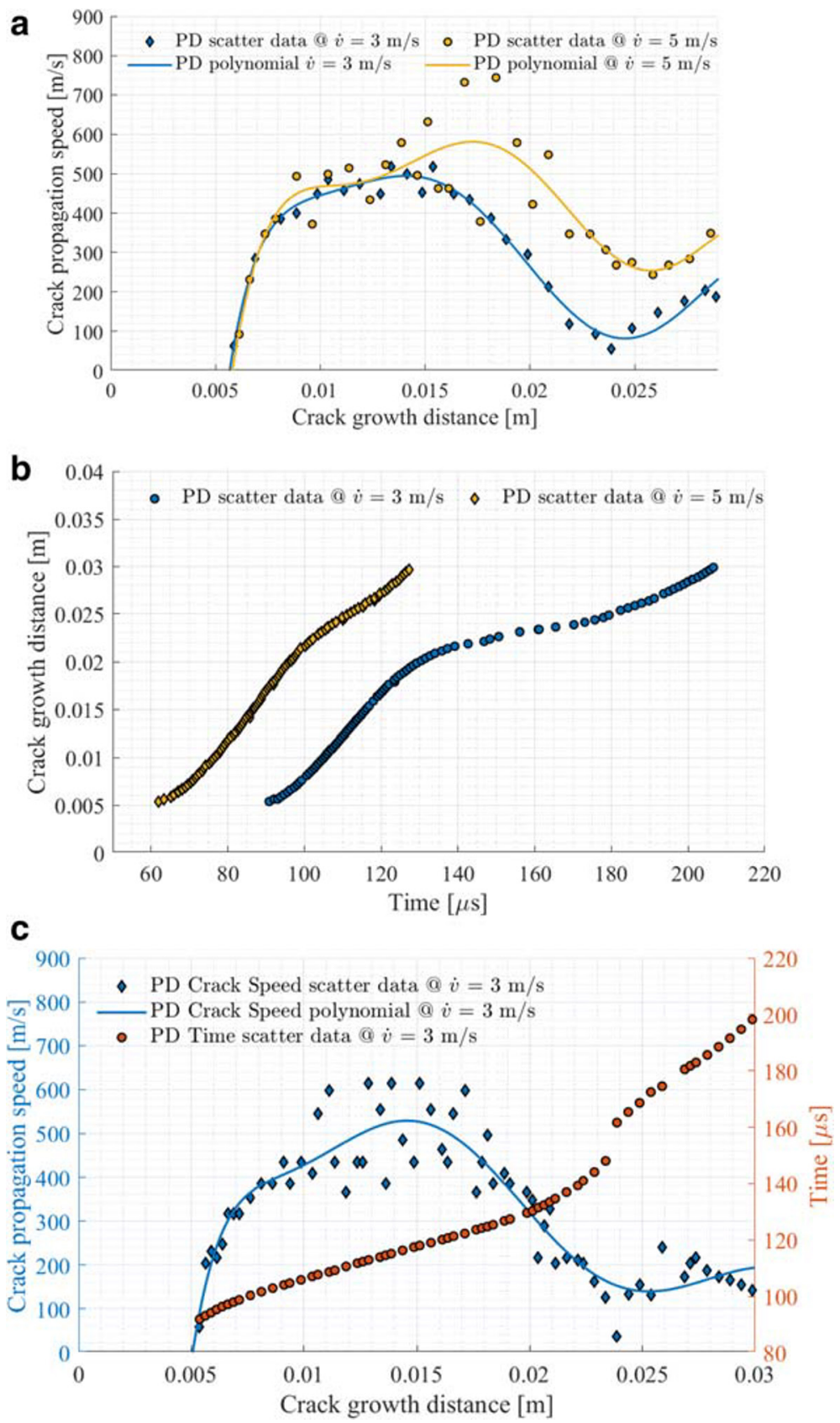

Fig. 7 PMMA plate with the hole radius of $R=0.005 \mathrm{~m}$ under velocity $\mathrm{BC}$ of $\dot{v}=3 \mathrm{~m} / \mathrm{s}$ and $\dot{v}=5 \mathrm{~m} / \mathrm{s}$ : a Variation of the crack propagation speed with increasing crack growth distance. $\mathbf{b}$ Variation of the crack length with time. $\mathbf{c}$ Variation of the crack propagation speed and time with increasing crack growth distance for PMMA plate with the hole radius of $R=0.003 \mathrm{~m}$ under velocity $\mathrm{BC}$ of $\dot{v}=3 \mathrm{~m} / \mathrm{s}$ 
propagation for lower velocity BC. Additionally, Fig. 7c shows the results of the crack propagation speed and time when velocity $\mathrm{BC}$ of $\dot{v}=3 \mathrm{~m} / \mathrm{s}$ is applied on a plate with a hole radius of $R=0.003 \mathrm{~m}$. In this case, the crack arrest is more clear and is around $13 \mu$ s starting at the time $t=148 \mu \mathrm{s}$ when the crack tip is at $d_{y}=0.023 \mathrm{~m}$. At the same moment, the crack propagation speed is dropping to $V=110 \mathrm{~m} / \mathrm{s}$ with further recovery. One of the reasons for the clear crack arrest period can be that the crack propagates as a straight line and deflects only towards the hole (Fig. 6a, $R=0.003 \mathrm{~m}$ ).

\subsection{Hole Defects in Concrete}

PD simulations of PMMA material showed good agreement with experimental tests for the crack propagation behaviour in the structure including a hole defect as described in "Section 3.2". Having this in mind, another type of brittle material, concrete, is considered for fracture analysis for the three-point-bending problem. The dimensions of the rectangular plate, the sizes and location of the defects are selected the same as described in "Section 3.2". The following material properties of the concrete are specified: Young's modulus of $E=$ $17 \mathrm{GPa}$, Poisson's ratio of $\nu=0.15$ and the energy release rate of $G_{0}=110 \mathrm{~J} / \mathrm{m}^{2}$. The PD models are discretised with 160,000 material points with uniform spacing between them $\Delta x=$ $0.00025 \mathrm{~m}$ and horizon size of $\delta=3.015 \Delta x$. Uniform time step size of $\Delta t=0.057 \mu \mathrm{s}$ is used.

For the application of the boundary conditions, the fictitious region approach is used, as shown in Fig. 3b. Eqs. 16 and 17 are used for specifying velocity BCs in the region $R_{f}$ and fully fixed BCs (Eqs. 14 and 15) representing the lower supports are applied in the area $R_{f_{s}}$. The concrete plate is subjected to three different velocity BCs: (a) $\dot{v}=0.5 \mathrm{~m} / \mathrm{s}$, (b) $\dot{v}=0.75$ $\mathrm{m} / \mathrm{s}$ and (c) $\dot{v}=1 \mathrm{~m} / \mathrm{s}$.

Figure 8 represents the damage maps for concrete plate subjected to the bending loading. Five cases are analysed with a varying radius of the hole defects: $R=0$, only pre-existing crack is allocated, $R=0.002 \mathrm{~m}, R=0.003 \mathrm{~m}, R=0.004 \mathrm{~m}, R=0.005 \mathrm{~m}$. The fracture behaviour of the concrete plates is similar to the PMMA material where the crack is curving towards the holes and increase in hole radius leads to an increase in curvature of the crack. Moreover, the existence of a hole in the plate influences the crack propagation speed and evaluating the crack speed at the location of the crack tip at $y=$ $d y=0.025 \mathrm{~m}$ (area of curvature) demonstrates a decrease in crack speeds with an increase in hole radius. The propagation speed of the crack towards the location of the applied velocity BC is presented in Fig. 9a. The arrest mechanism in the concrete structure with the presence of the holes ahead of the propagating crack can be noticed (Fig. 9b). The period of the crack arrest in Fig. 9b is very small and after the crack propagates away from the hole, the crack propagation speeds are even higher than the crack speeds before the crack arrest. For all simulations, a constant increase in crack propagation speed is observed first. However, as the crack approaches closer to the hole approximately $0.01 \mathrm{~m}$ behind the location of the hole, i.e. the crack tip is at $x=0 \mathrm{~m}, y=$ $\left(d_{y}-0.01\right) \mathrm{m}$, leads to the curvature of the main crack and a gradual drop of speed on average by $70 \%$.

Numerical results utilizing PD showed a similar pattern of brittle fracture with the existence of the holes in the pre-cracked plate under three-point-bending conditions. The crack is propagating very fast towards the location of the load application with the crack propagating speeds reaching up to $V=600 \mathrm{~m} / \mathrm{s}$ for the load cases of $\dot{v}=5 \mathrm{~m} / \mathrm{s}$ for PMMA and $\dot{v}=1 \mathrm{~m} / \mathrm{s}$ 

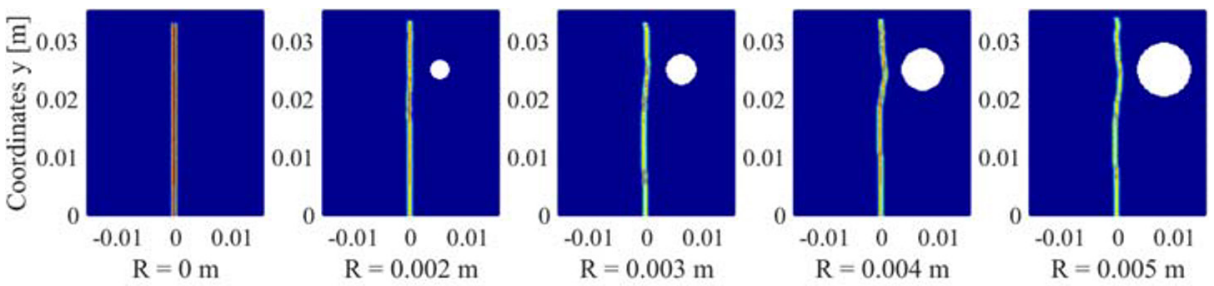

(a)
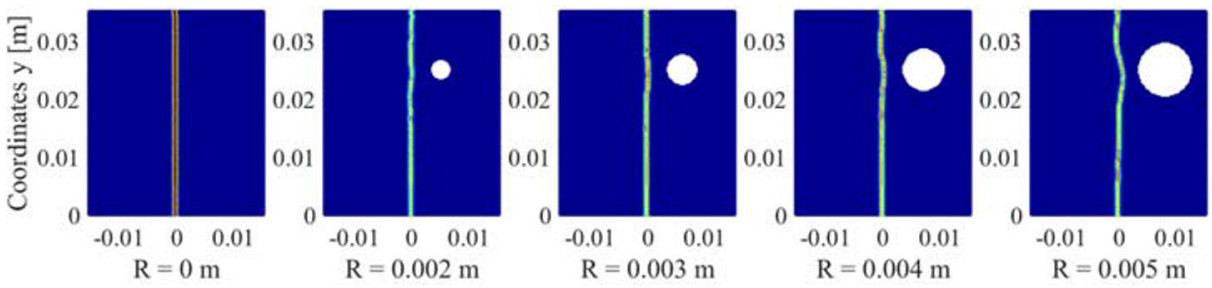

(b)
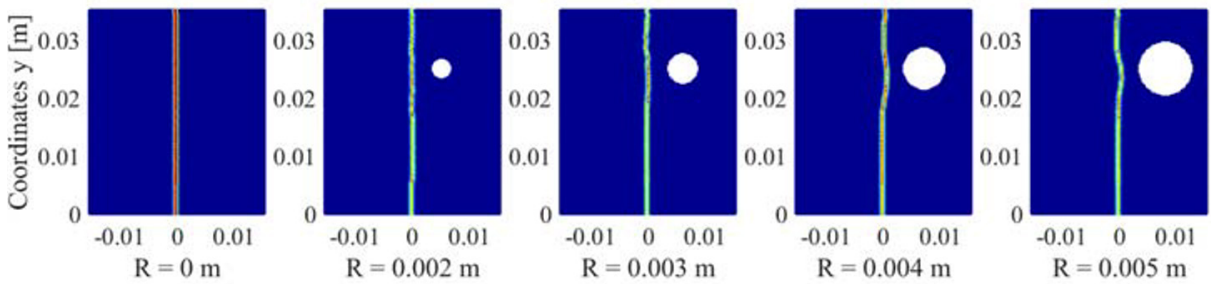

(c)

Fig. 8 Damage maps of the concrete plate with different values of the hole radius $R$ under velocity BC: a $\dot{v}=0$ $.5 \mathrm{~m} / \mathrm{s}$ at time $t=485.56 \mathrm{~s}, \mathbf{b} \dot{v}=0.75 \mathrm{~m} / \mathrm{s}$ at $t=285.63 \mathrm{~s}, \mathbf{c} \dot{v}=1 \mathrm{~m} / \mathrm{s}$ at $t=228.5 \mathrm{~s}$

for a concrete plate. On the other hand, more brittle material as concrete showed faster crack propagation under lower velocity boundary conditions.

\section{Influence of Micro-cracks on Crack Propagation in a Plate Under Tension Loading}

In this section, the impact of the micro-cracks on the main (macro-) crack behaviour is analysed. The geometric parameters characterising the interaction of the macro- and microcracks are the dimensions of the cracks, distances between the micro-cracks and the orientation of the micro-cracks.

The PD model consists of a macro-crack with a size of $a=0.045 \mathrm{~m}$ at the left side of the plate (Fig. 10a). Five different cases of distributed micro-cracks are considered with a length of $a_{c} / a=0.1$. The distances between the micro-cracks are $a / s$ and $a / r$ with $s=r=3$, and they are located in front and/or around the macro-crack tip, as shown in Fig. 11. For each of the cases from (a) to (e), micro-crack configurations are analysed with the crack inclination of $\alpha$ varying 


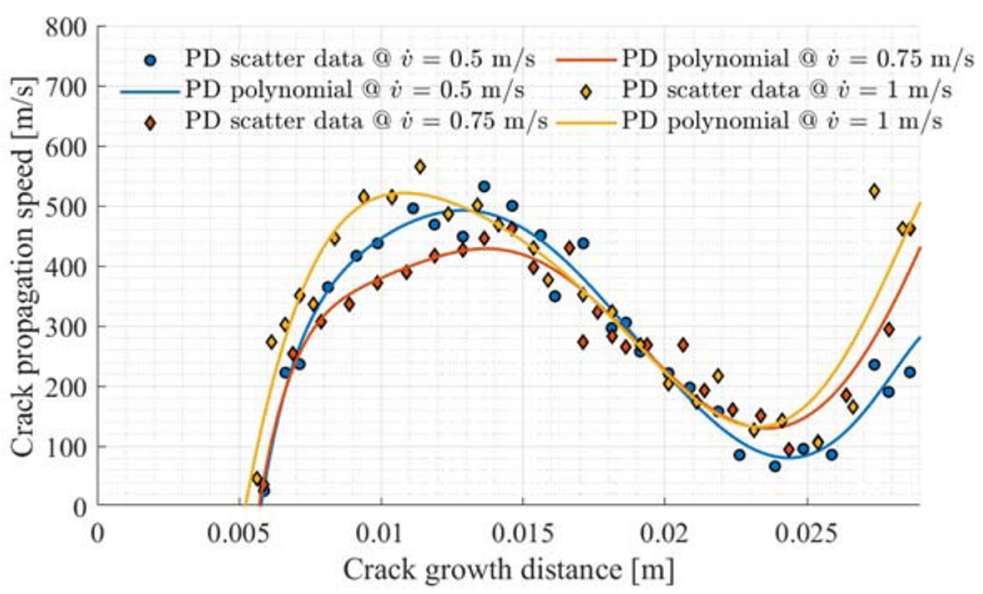

(a)

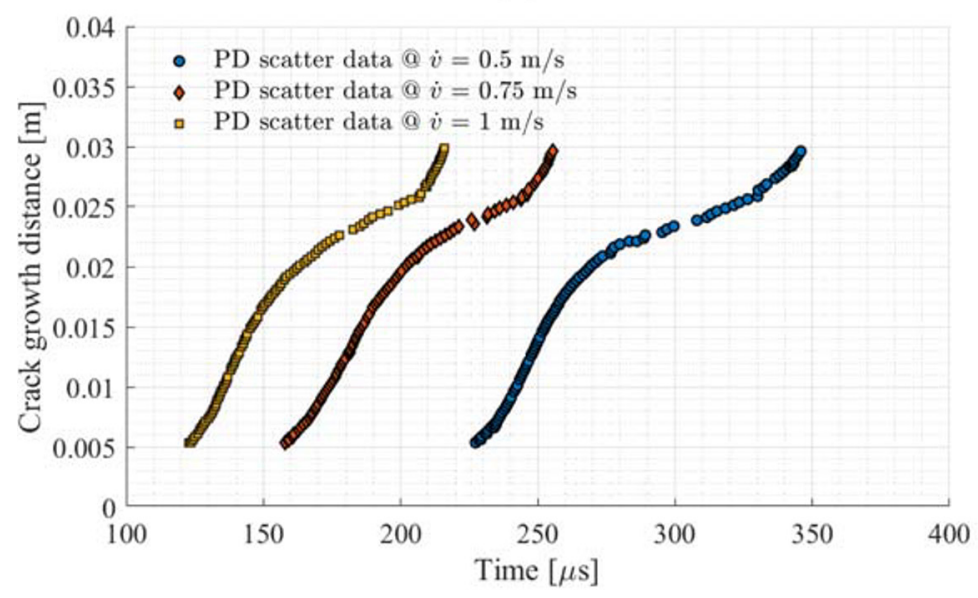

(b)

Fig. 9 Concrete plate with the hole radius of $R=0.005 \mathrm{~m}$ under different velocity BC: a Variation of the crack propagation speed with increasing crack growth distance. b Variation of the crack length with time

from $0^{\circ}$ to $90^{\circ}$. Micro-cracks are regularly located in $+\mathrm{y}$ and/or $-\mathrm{y}$ domains and/or in front of the main crack tip.

The geometric parameters of the concrete plate are selected as the length of $L=0.22 \mathrm{~m}$, width of $W=0.31 \mathrm{~m}$ and thickness of $h=0.003 \mathrm{~m}$ with material properties Young's modulus of $E=17 \mathrm{GPa}$ and Poisson's ratio of $\nu=0.15$.

PD simulations are performed under an explicit time integration scheme with a stable time step size of $\Delta t=1$ for quasi-static problems ("Section 4.1") by means of the adaptive relaxation technique [41]. For dynamic PD simulations, $\Delta t=0.2 \mu \mathrm{s}$ is used [40] in "Section 4.2". The stability criterion for concrete plate in "Sections 3.2 and 4.2" differs because of the dependency on horizon size $\delta=3.015 \Delta x$ and spacing between the points is selected as $\Delta x=0.0005 \mathrm{~m}$, instead of $\Delta x=0.00025 \mathrm{~m}$. 


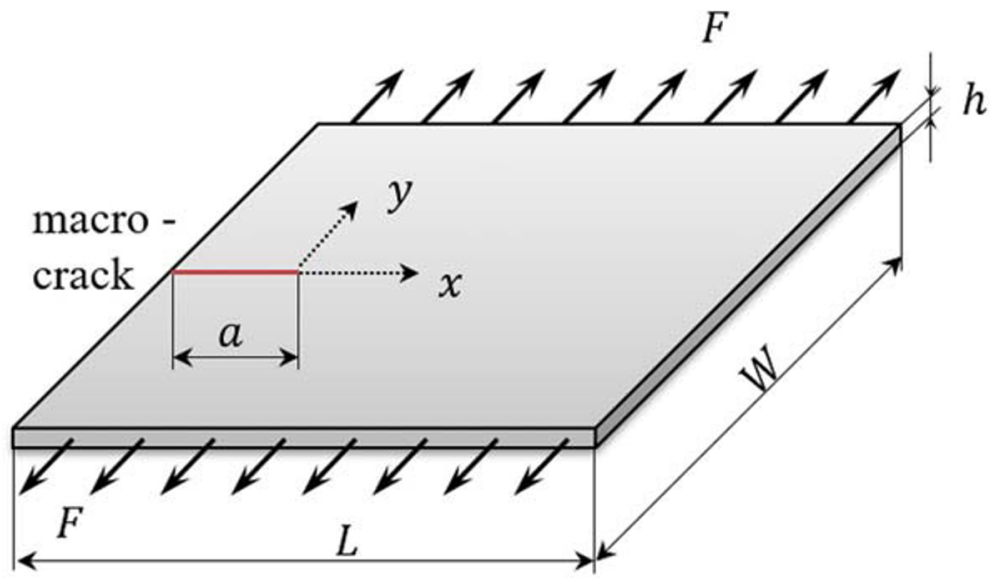

(a)

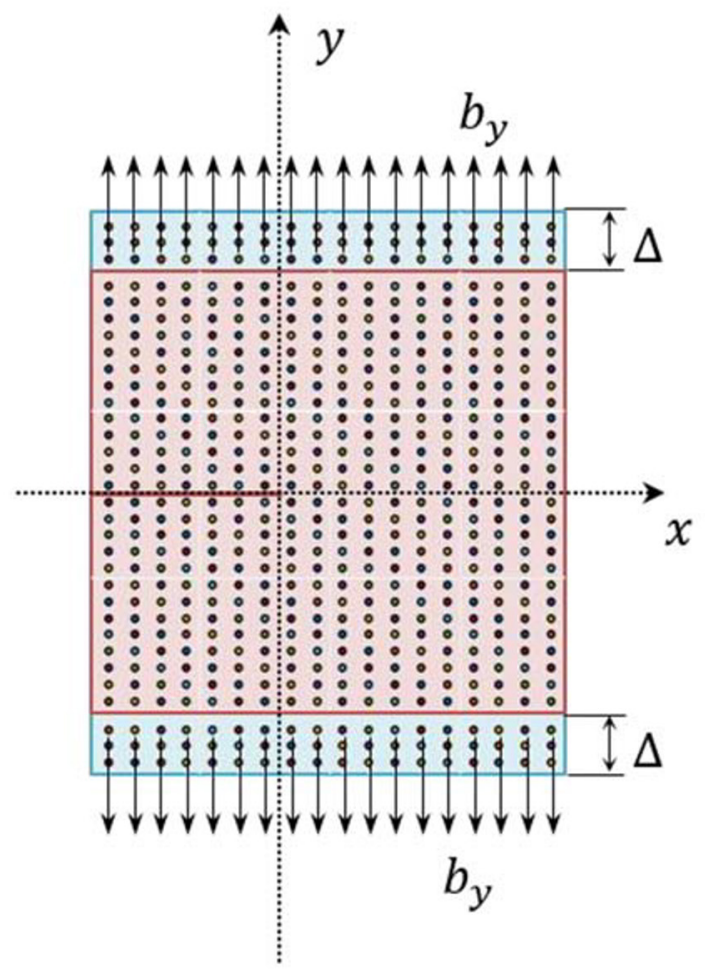

(b)

Fig. 10 Sample geometry: a square plate with a macro-crack under quasi-static loading; $\mathbf{b}$ spatial discretisation of the square plate with a macro-crack under body force density boundary conditions 


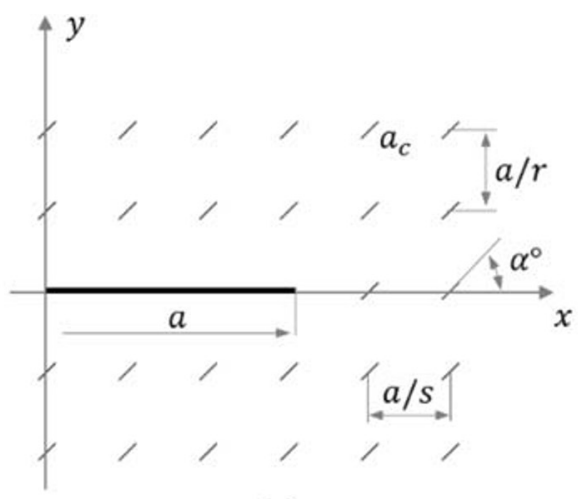

(a)

(b)

(d) (c)

(e)

Fig. 11 Micro-crack distribution diagrams: a micro-cracks surrounding the main crack tip; b micro-cracks ahead of the crack tip; $\mathbf{c}$ asymmetrical arrangement of micro-cracks (+y domain) ahead of the main crack tip; $\mathbf{d}$ a set of micro-cracks ahead of the crack tip; e single micro-crack ahead of the main crack tip

\subsection{Critical Load Evaluation for a Plate-Containing Micro-Cracks}

The purpose of this section is to investigate if the presence of the defects ahead of the macrocrack tip facilitates its further propagation. In order to achieve this, five micro-crack distribution cases (Fig. 11) are considered. The plate is loaded symmetrically by applying quasi-static tensile loading in the $y$-direction (Fig. 10a). Based on the convergence study, with an applied time step size of $\Delta t=1 \mathrm{~s}$, a total time step of 3000 is sufficient to reach the quasi-static loading condition by means of adaptive dynamic relaxation (ADR) method [41]. After applying an initial loading of $F=90 \mathrm{~N}$ and reaching the steady-state solution the applied force is increased by $\Delta F=1 \mathrm{~N}$ for each 3000 time steps. The stretch of the bonds between material points is evaluated and when the first bond is broken if the stretch exceeds the critical stretch, i.e. $s>s_{c}$, the applied force is referred to as the critical load $p$. The critical loads are identified as $p^{0}$ for a 
plate with only single macro-crack and $p^{*}$ for the plate with a macro-crack and distributed micro-cracks.

The PD model is discretised with 69,520 material points (Fig. 10b) with uniform spacing between them $\Delta x=0.001 \mathrm{~m}$ and $\delta=3.015 \Delta x$. A quasi-static loading is applied as a body force density:

$$
b_{y}=\frac{F}{\Delta V_{\Delta}}
$$

where $\Delta V_{\Delta}$ is the volume of the boundary layer, $\Delta V_{\Delta}=1.98 \times 10^{-6} \mathrm{~m}^{3}$.

The PD numerical models are analysed based on the relationship between the SIF of the case with a macro-crack $K_{0}$ and cases including micro-cracks $K^{*}$ for all PD numerical models. First, constant fracture energy of $G_{0}=570 \mathrm{~J} / \mathrm{m}^{2}$ is considered for all cases. In this respect, the numerical model is evaluated under constant critical stretch-based criteria, $s_{c}^{*}=s_{c}=0.0038$ for all five cases of micro-crack distributions with $s_{c}^{*}$, as well as for the case with only one macro-crack with $s_{c}$. Furthermore, critical stretch is not influenced by micro-crack configurations and crack inclination angles $\alpha$ varying from $0^{\circ}$ to $90^{\circ}$.

The results of the PD simulations are presented in Fig. 12 based on the ratio of the critical loads $p^{*}$ and $p^{0}$. Although a similar pattern of the micro-crack influence on main crack propagation is noticed by analytical solutions [17], analytical results have lower ratios of the critical loads for the inclination angles from $0^{\circ}$ to $45^{\circ}$ compared to the PD simulations. The reason for the difference in the critical loads is the critical stretch which is constant in all PD simulations. The authors in [44] illustrate that the stress intensity factor is decreasing when the main crack is surrounded by multiple micro-cracks. Furthermore, Kachanov $[11,14]$ showed the influence of the micro-crack arrays and the inclination of the cracks on the geometry, which results in shielding effects or weakening.

Kachanov [14] evaluated two configurations of the micro-crack distributions: collinear cracks and stacked ones. He demonstrated in his work that the collinear cracks showed the stress amplification effect, which leads to an increase of SIF; contrary, the stacked

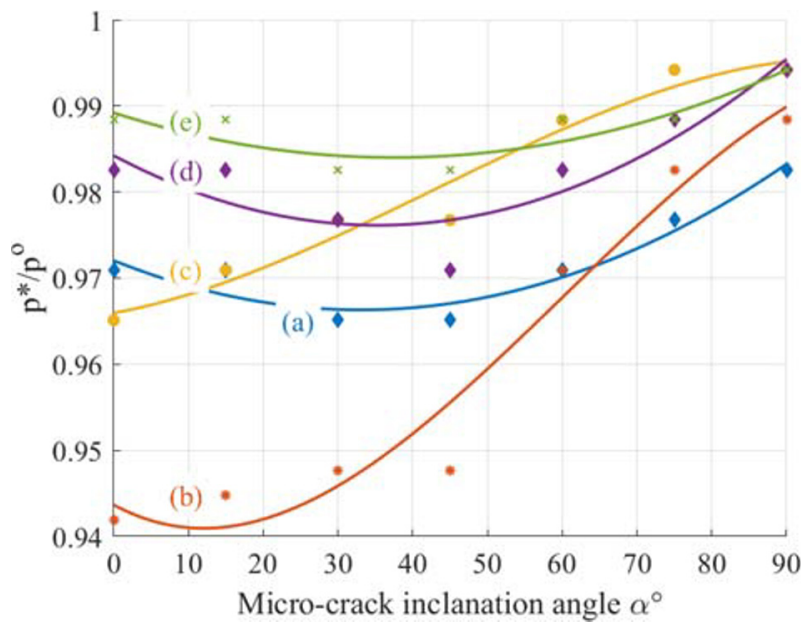

Fig. 12 The ratios of the critical loads as a function of micro-crack orientation when constant critical stretch is considered for micro-crack distribution cases from (a) to (e). Markers present the PD scatter data and solid lines-PD polynomials for cases from (a) to (e) 
configurations showed the stress shielding effect with the decrease of SIF. The further evaluation of the distributed cracks by Kachanov showed the influence of the crack dimensions and distances on the amplifying or shielding outcome and depending on the crack distributions this two phenomena can compete. The author states that for traction BC (mode I), if the distances between the collinear $a / s$ and stacked $a / r$ configurations are higher than the length $a_{c}$ of the cracks, the shielding effect is dominant. In order to get the amplifying effect of the cracks in the structure, the distance $a / s$ should be much smaller than $a / r$ [14]. In addition, other factors have an influence on the SIF, such as the crack number in the periodic coplanar row of cracks, periodic stack of parallel cracks and the rotation of the cracks. According to Kachanov, with the increased number of parallel stack cracks the SIF is decreasing, instead of for the coplanar row of cracks, SIF is increasing and $K^{*} / K_{0} \approx 1$. Moreover, the distribution of the micro-cracks around the main crack brings stress shielding effect. Similar effects of the arrays on the macro-crack tip, causing SIF amplification is found by multiple authors $[15,45$, 46]. When the micro-cracks are rotated by $90^{\circ} \geq \alpha>0$, high impact is noticed of micro-cracks on macro-crack. The surrounding distribution of the micro-cracks leads to increase of SIF with the micro-crack rotation from $0^{\circ}$ to $90^{\circ}$.

Evaluating the selected micro-crack distribution diagrams for PD analysis, the distances between the micro-cracks are bigger then the length of the cracks $a / s \gg a_{c}$ and the effect of the shielding are dominant in the structure. Thus, SIF for the cases, including micro-cracks should be smaller than the cases with only one macro-crack, resulting $K^{*}<K_{0}$. Considering the outcome of the analytical solutions from [14] for the impact of the micro-cracks with rotational angles $\alpha=0$ degrees on SIF of the macro-crack tip, the following relationships $K^{*} / K_{0}$ are considered in the PD model: (a) $K^{*} / K_{0}=0.90$, (b) $K^{*} / K_{0}=0.84$, (c) $K^{*} / K_{0}=0.93$, (d) $K^{*} / K_{0}=$ 0.96, (e) $K^{*} / K_{0}=0.98$.

The selected $K^{*} / K_{0}$ are implemented in the numerical model by factorising the critical stretch values by $K^{*} / K_{0}$ ratios, where $s_{c}^{*}<s_{c}$. Furthermore, to include in PD model the influence of the micro-crack inclinations on a macro-crack tip, critical stretch ratios are gradually increasing, resulting for the cases at $\alpha=90 \mathrm{deg}$ : (a)-(c) $s_{c}^{*} \approx 0.0036$, (d) $-\left(\right.$ e) $s_{c}^{*} \approx s_{c}=0.0038$. The difference in the applied critical stretch values between the cases (a)-(c) and (d)-(e) is resulted by the location of the micro-cracks, as the micro-crack distribution behind and above the macro-crack tip with the angle of rotation $\alpha=90^{\circ}$ leads to $K^{*} / K_{0}<0.96$ [14]. The employment of the relationship of $K^{*} / K_{0}$ in the PD model (Fig. 13) showed more reasonable results compared to the analytical solution [17] which confirms the dependency of the crack propagation on micro-crack distribution and deviation angle.

\subsection{Crack Propagation Behaviour in a Plate-Containing Micro-cracks}

According to the study in "Section 4.1", the influence of the micro-cracks on SIF of the macrocrack tip and their impact on the critical load is numerically demonstrated. The interest of this section is to evaluate the influence of the micro-cracks on macro-crack propagation behaviour and speed. To calculate the crack propagation speeds, the concrete plate in Fig. 14a is subjected to dynamic BCs and loaded symmetrically by applying velocity constraints of $v^{*}=0.4 \mathrm{~m} / \mathrm{s}$ on the plate edges at $y={ }^{W} / 2$ and $y=-{ }^{W} / 2$. The boundary conditions, proposed in [47], is applied to the fictitious boundary layer $R_{f}$. The size of the $R_{f}$ region is equal to the horizon size, which is $\delta=3.015 \Delta x$. The BCs are enforced as follows: 


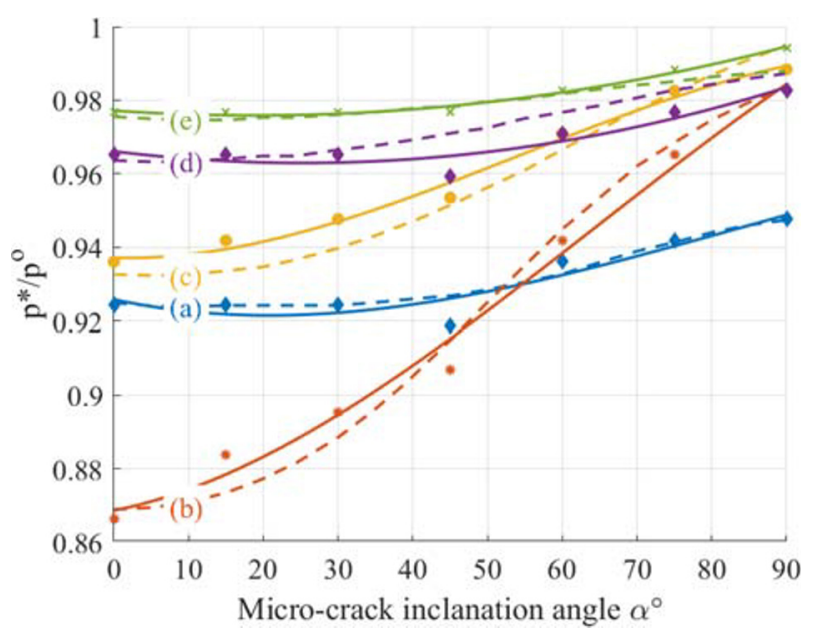

Fig. 13 The ratios of the critical loads as a function of micro-crack orientation when $K^{*} / K_{0}$ for micro-crack distribution cases: (a) $K^{*} / K_{0}=0.90$, b $K^{*} / K_{0}=0.84$, (c) $K^{*} / K_{0}=0.93$, (d) $K^{*} / K_{0}=0.96$, (e) $K^{*} / K_{0}=0.98$. Markers present the PD scatter data, dashed lines_-PD polynomials for cases from (a) to (e), and solid lines - analytical solution for the cases from (a) to (e)

$$
\begin{gathered}
u_{f}\left(x_{f}, y_{f}, t+\Delta t\right)=u(x, y, t) \text { with } y_{f} \in R_{f\left(\mathrm{y}=\frac{W}{2}\right)} \\
v_{f}\left(x_{f}, y_{f}, t+\Delta t\right)=2 v^{*}\left(x, \mathrm{y}=\frac{W}{2}, t+\Delta t\right) \times t-v(x, y, t) \\
u_{f}\left(x_{f}, y_{f}, t+\Delta t\right)=u(x, y, t) \text { with } y_{f} \in R_{f\left(\mathrm{y}=-\frac{W}{2}\right)} \\
v_{f}\left(x_{f}, y_{f}, t+\Delta t\right)=2 v^{*}\left(x, \mathrm{y}=-\frac{W}{2}, t+\Delta t\right) \times t-v(x, y, t)
\end{gathered}
$$

The PD model in Fig. 14b is discretised with 272,800 material points with uniform spacing between them $\Delta x=0.0005 \mathrm{~m}$. All simulations are performed with a time step size of $\Delta t=$ $0.2 \mu \mathrm{s}$ and stopped at $t=300 \mu \mathrm{s}$.

In "Section 4.1", it is noted that factorising the critical stretch values for the cases (a)-(e) with micro-cracks distribution in the plate provided the results of the ratios of critical loads which are very close to the analytical solutions. Another important point is to see if the SIF at the macro-crack tip influences the crack propagation behaviour and speeds. It should be noted that the relation between $K^{*} / K_{0}$ is presenting the influence of the micro-cracks on the SIF of the macro-crack tip and is not speed dependent. This means that constant fracture energy model is used and critical fracture energy is not changing with the crack propagation speed [48, 49].

Firstly, the PD numerical models for the cases (a)-(e) in Fig. 11 are analysed with the relation between the SIF of the case with macro-crack $K_{0}$ and the cases including micro-cracks 


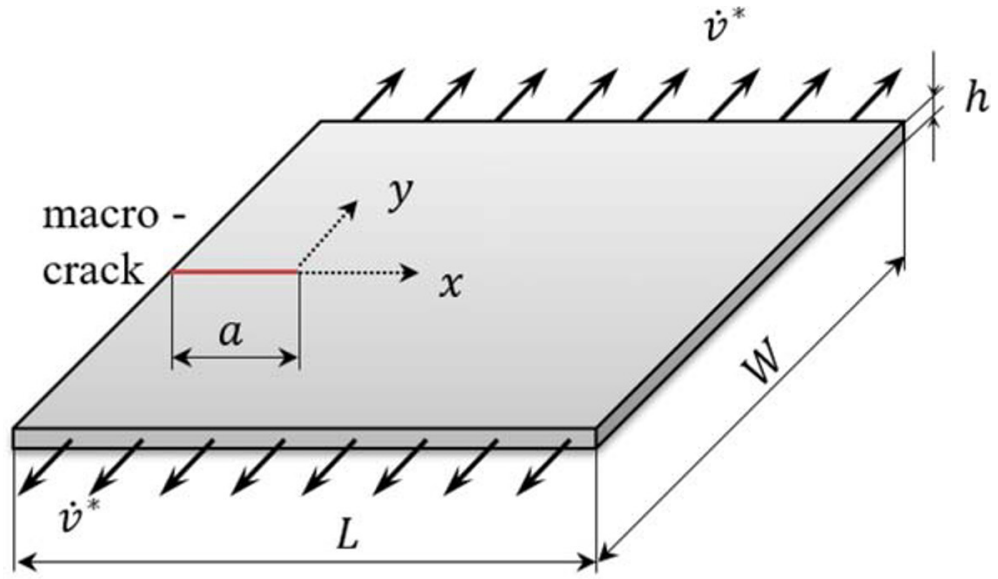

(a)

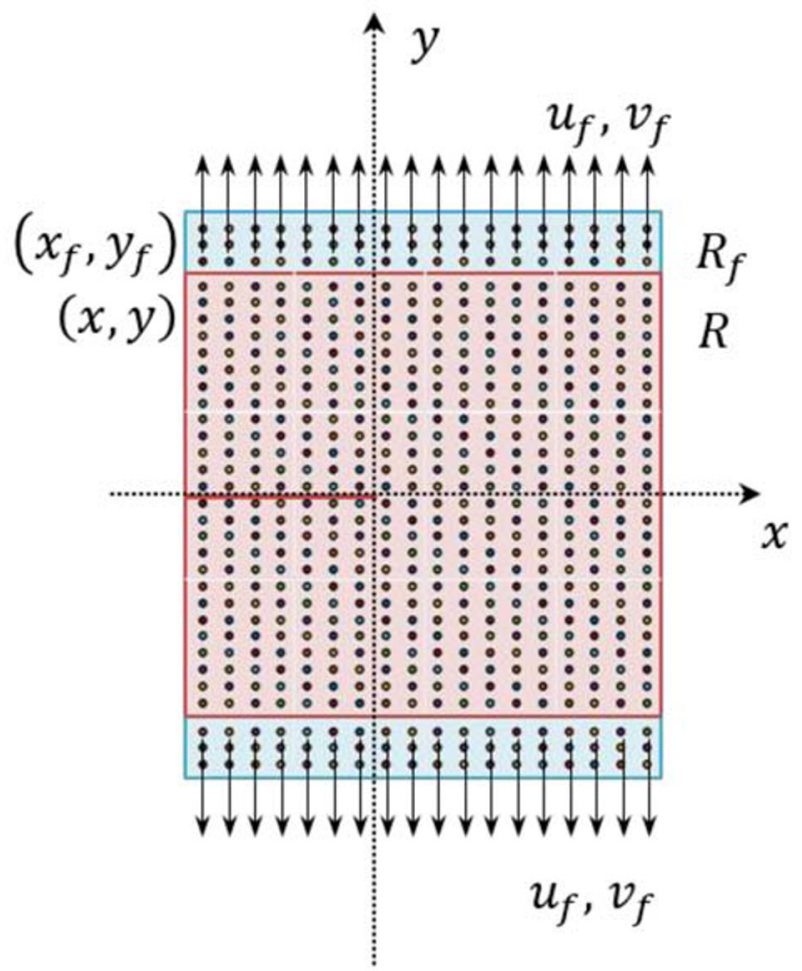

(b)

Fig. 14 Sample geometry: a square plate with a macro-crack under velocity BCs; $\mathbf{b}$ spatial discretisation of the square plate with a side macro-crack under imposed BCs 
$K^{*}$ by using a constant critical stretch. Secondly, the relation between $K^{*} / K_{0}$ is varied in the PD model according to the cases: (a) $K^{*} / K_{0}=0.90$, (b) $K^{*} / K_{0}=0.84$, (c) $K^{*} / K_{0}=0.93$, (d) $K^{*} / K_{0}=$ 0.96 , (e) $K^{*} / K_{0}=0.98$. The relations $K^{*} / K_{0}$ are implemented in the numerical model in the same way as described in the "Sect 4.1:" by factorising the critical stretch values by $K^{*} / K_{0}$ ratios.

Figure 15 presents the damage plots for all cases when constant critical stretch is considered and Fig. 16 is for $K^{*} / K_{0} \neq 1$ and the micro-crack inclination angles are $\alpha=$ $0^{\circ}, 45^{\circ}$ and $90^{\circ}$. It can be noted that the micro-crack inclination angles have an influence on the macro-crack propagation behaviour where the macro-crack tends to follow the orientation of the micro-cracks. Moreover, when the micro-crack is located at the front and perpendicular to the macro crack, $\alpha=90^{\circ}$, this leads to bifurcation of the macro-crack. Both types of critical stretch implementation showed a similar pattern of the crack propagation, but the difference in the crack propagation and speeds can be noticed in Figs. 17 and 18. The adjusted critical stretch value according to SIF cases show higher crack propagation speeds as in Fig. 18.

The crack propagation speed is evaluated by Eq. 20, when the macro-crack tip location becomes $x=0.05 \mathrm{~m}$. From Figures 17 and 18 it can be noticed that the microcracks parallel to the macro-crack leads to an increase in crack propagation speed. On the other hand, the micro-cracks perpendicular to the macro-crack yields shielding effect [35] leading to decrease in crack propagation. Moreover, the crack opening of surrounding micro-cracks leads to energy dissipation and increase in crack propagation resistance. When the micro-cracks are not distributed in front of the macro-crack as in case (c), the crack propagation speed is similar to the case with only macro-crack is allocated. The micro-cracks act as the suppressors of the macro-crack propagation in the case (a) of micro-crack distribution (inclination $\alpha=0^{\circ}$ ) which decreases the crack speed compared to the case (b).

The other point to mention is the crack propagation over time. Figure 19 presents results for each case (a) to (e) of micro-crack distributions with inclination angles $\alpha$ varying from $0^{\circ}$ to $90^{\circ}$ ahead of the main crack tip when the relation $K^{*} / K_{0} \neq 1$ is implemented. The numerical results show that only collinear distribution of the microcracks, case (d) and (e), in front of the macro-crack delaying the crack propagation at the initial stage. Moreover, for most of the studied cases, the bigger crack inclination angle increases the propagation time. On the other hand, the hold off time is very small, around $30 \mu \mathrm{s}$, and after pathing through the micro-crack, the crack continues fast propagation. Only the crack bifurcation in all the cases showed major impact on crack delay. Based on the performed PD simulation results, it can be concluded that the types of micro-cracks, their sizes, distributions and orientations have a remarkable influence on the toughening mechanisms in the system.

\section{Conclusion}

This study presents the application of ordinary state-based PD model on complex hole defect crack, macro- and micro-crack interaction problems. The results demonstrate that PD numerical model captures the fracture phenomena in concrete material including crack evolution and propagation. It is presented that the defect in the structure plays a significant role on the structural behaviour and the crack propagation path. The results of 

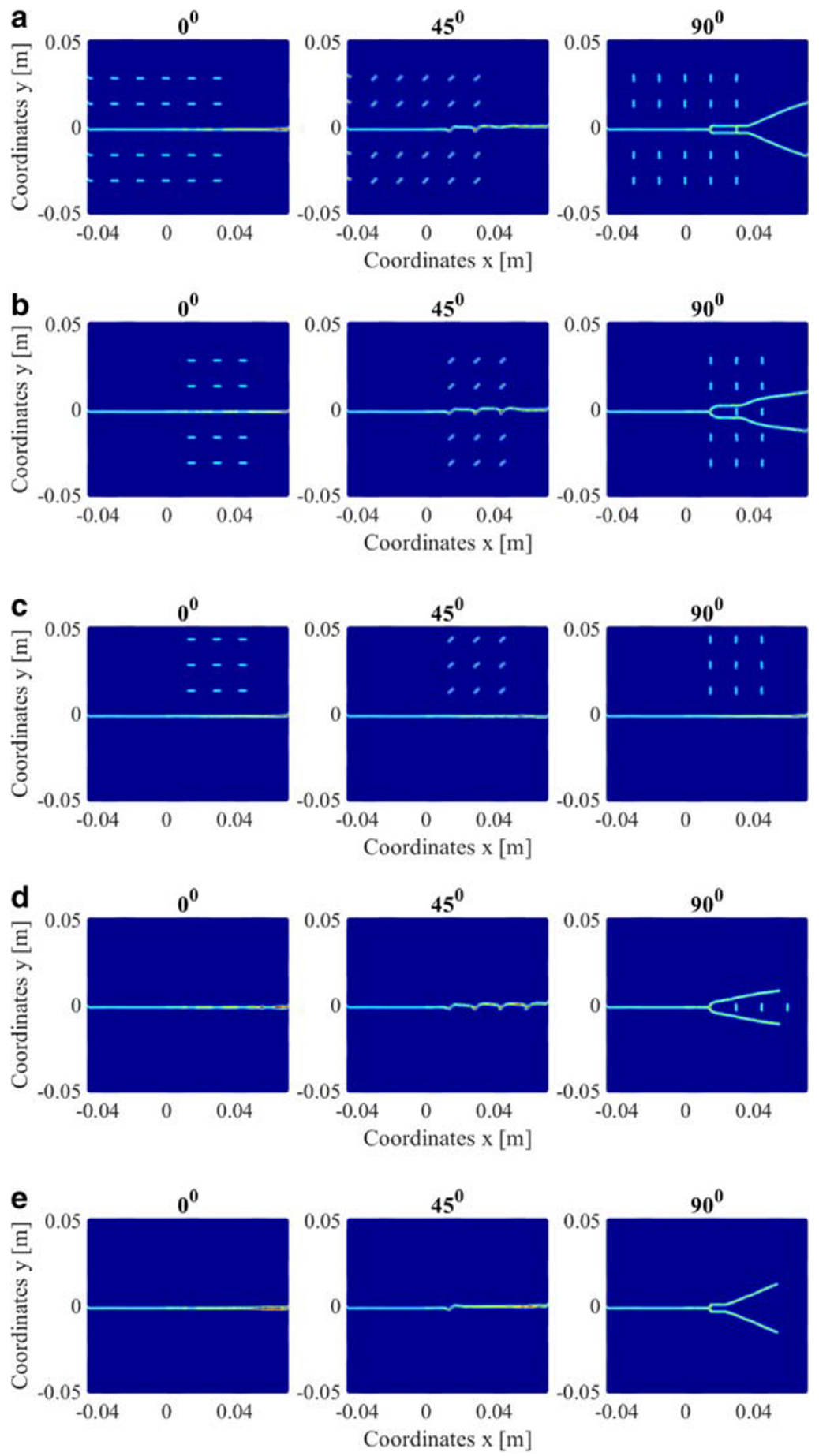

Fig. 15 Damage plots of the crack propagation, when constant critical stretch is considered for the cases from a to e with multiple micro-cracks with the crack inclination of $\alpha=0^{\circ}, 45^{\circ}$ and $90^{\circ}$ 

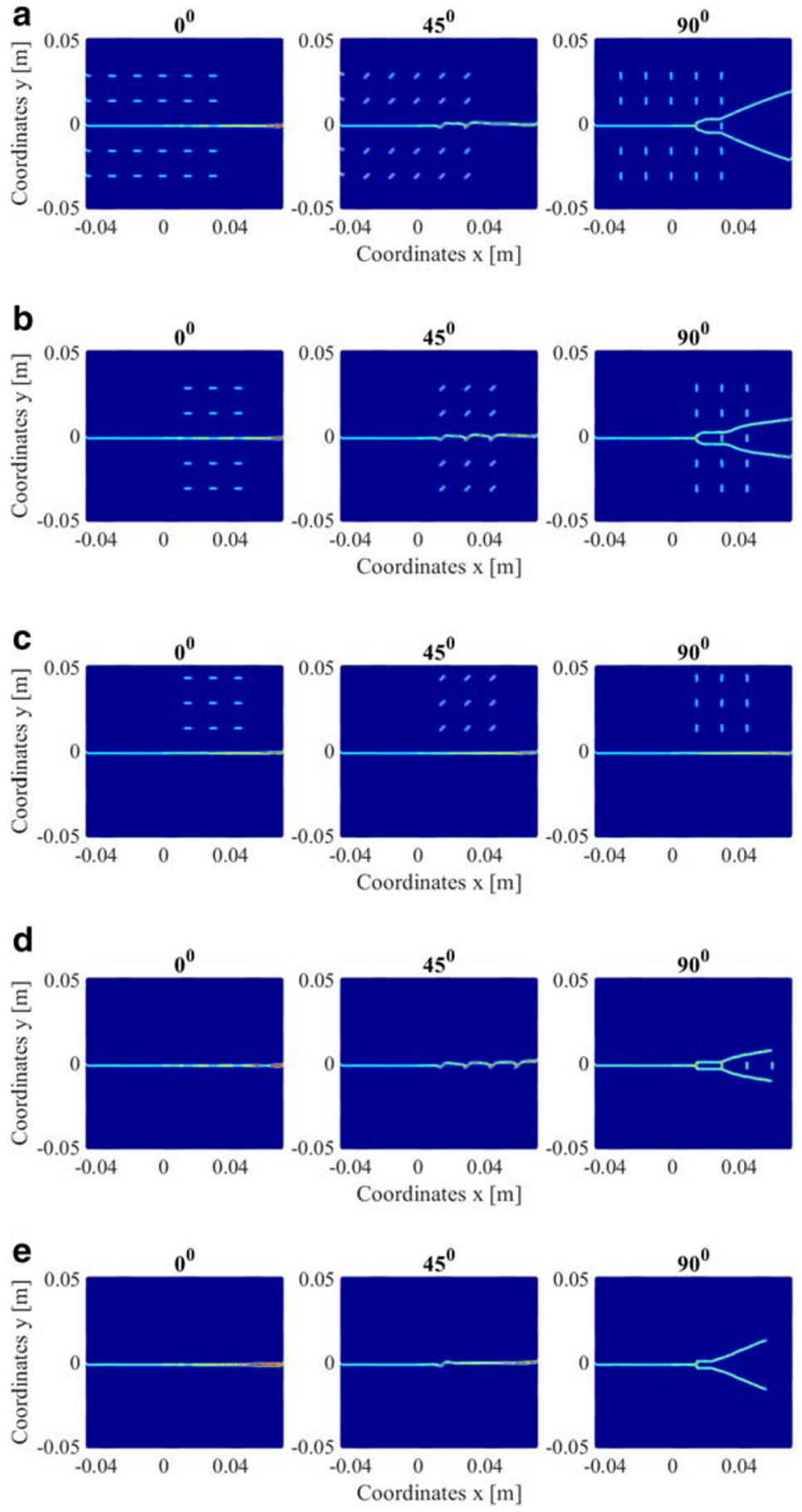

Fig. 16 Damage plots of the crack propagation for the cases from a to e when $K^{*} / K_{0}$ for micro-crack distribution: a $K^{*} / K_{0}=0.90$, b $K^{*} / K_{0}=0.84$, c $K^{*} / K_{0}=0.93$, d $K^{*} / K_{0}=0.96$, e $K^{*} / K_{0}=0.98$, with the crack inclination of $\alpha=$ $0^{\circ}, 45^{\circ}$ and $90^{\circ}$ 


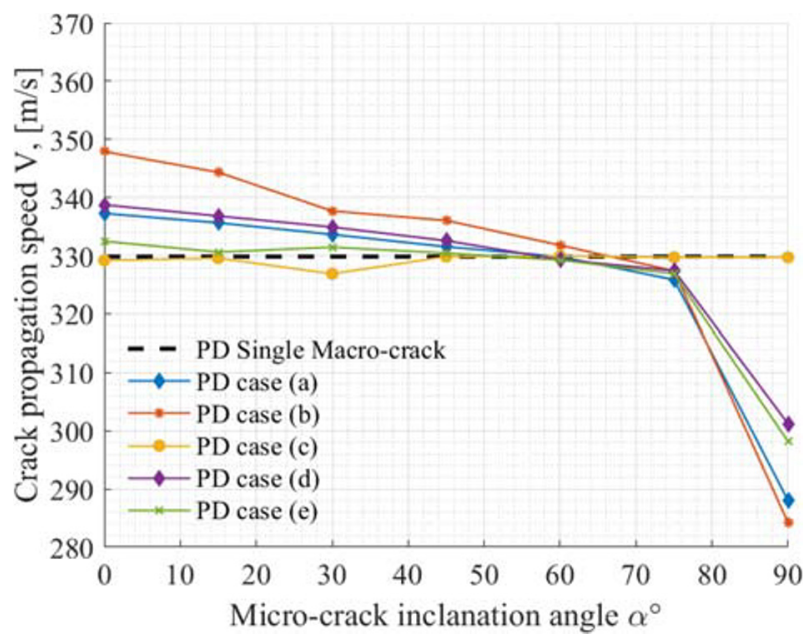

Fig. 17 Numerical results for the concrete plate under velocity $\mathrm{BC} v_{l}=0.4 \mathrm{~m} / \mathrm{s}$ for a crack speed as a function of micro-crack orientation and when constant critical stretch is considered. Crack speed is evaluated at the crack tip location $x=0.05 \mathrm{~m}$

the PD numerical model of the three-point-bending of the pre-cracked plate with a hole defect show that the crack arrest phenomena with the decrease of the crack propagation speed by $70 \%$ occurs when the crack deflects towards the hole. However, shortly after, the crack restores its original trajectory with an increase in speed. The increase in velocity boundary condition leads to larger deflections of the crack towards the hole. Similar characteristics were observed during the experiments which show the reliability of the performed simulations by means of PD numerical model.

The numerical results for the critical load in macro- and micro-crack interaction problem showed remarkable results compared to analytical solutions. PD model accurately captured the

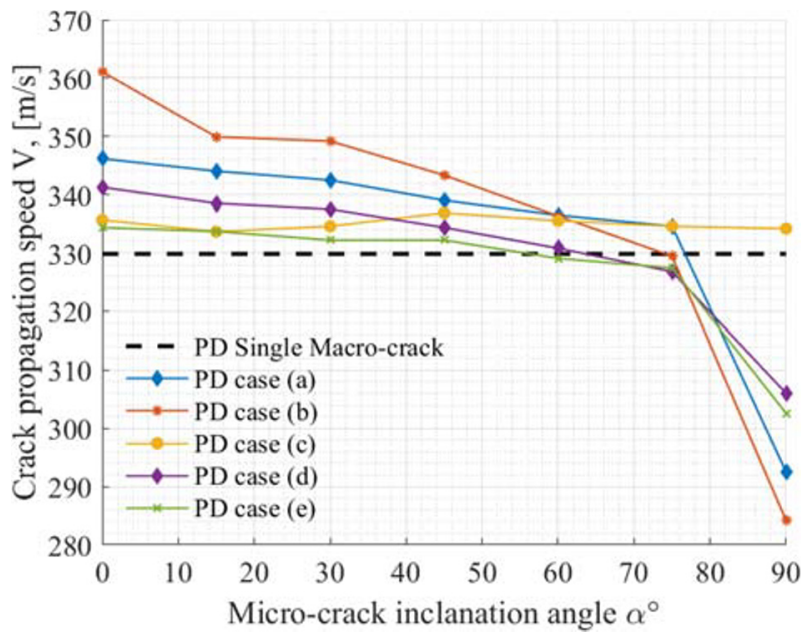

Fig. 18 Numerical results for the concrete plate under velocity BC $v_{l}=0.4 \mathrm{~m} / \mathrm{s}$ for a crack speed as a function of micro-crack orientation. The parameter $K^{*} / K_{0} \neq 1$ : a $K^{*} / K_{0}=0.90$, b $K^{*} / K_{0}=0.84$, c $K^{*} / K_{0}=0.93$, d $K^{*} / K_{0}=$ 0.96 , e $K^{*} / K_{0}=0.98$. Crack speed is evaluated at the crack tip location $x=0.05 \mathrm{~m}$ 

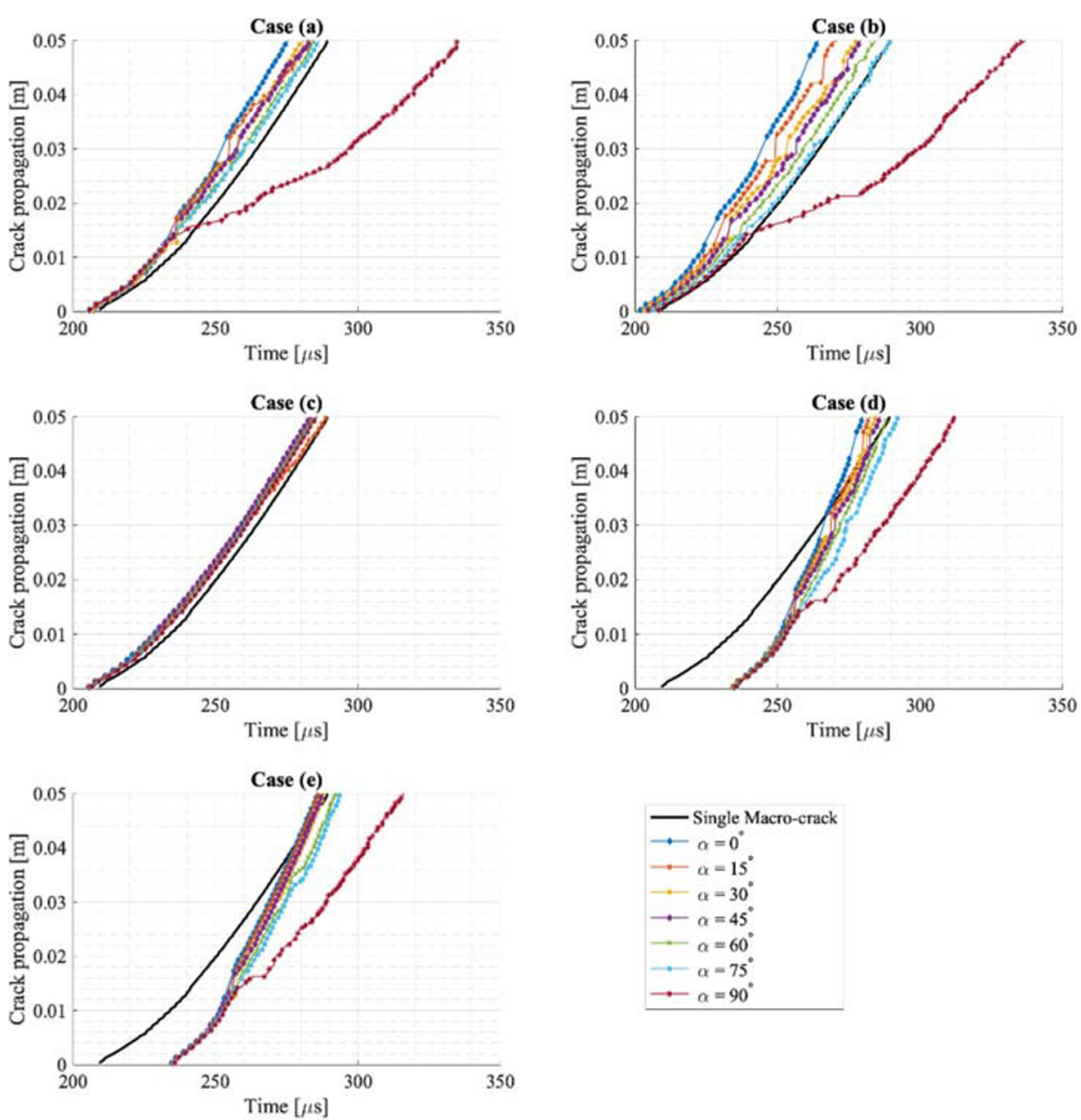

Fig. 19 Numerical results for the concrete plate under velocity BC $v_{l}=0.4 \mathrm{~m} / \mathrm{s}$ for a crack propagation as a function of time for all the cases (a) to (e) of the micro-crack distribution with different micro-crack inclination angles $\alpha$. The parameter $K^{*} / K_{0} \neq 1$ for cases: a $K^{*} / K_{0}=0.90, \mathbf{b} K^{*} / K_{0}=0.84$, c $K^{*} / K_{0}=0.93$, d $K^{*} / K_{0}=0.96$, e $K^{*} / K_{0}=0.98$

influence of the micro-crack inclination angles on the critical loads that a plate can withstand. It is concluded that the application of the parameter $K^{*} / K_{0}$ has a substantial impact on the results, as stress intensity factor is dependent on crack distribution and inclination angles. The application of the updated $K^{*} / K_{0} \neq 1$ for different micro-crack distributions showed close agreement with the analytical solutions. It can be noticed that the allocation of the microcracks in front of the macro-crack facilitates further propagation of the crack. Moreover, the macro-crack has a tendency to follow the micro-cracks' orientation, and in the case of the perpendicular, orientation of micro-crack with respect to the macro-crack leads to a further bifurcation. Analysing the crack propagation speeds showed that in the presence of the microcracks ahead of the macro-crack, the shielding effect is present, and the crack speeds drop by approximately $10 \%$. On the other hand, the parallel distribution of micro-cracks increases the crack speeds by approximately $6 \%$. 
Open Access This article is licensed under a Creative Commons Attribution 4.0 International License, which permits use, sharing, adaptation, distribution and reproduction in any medium or format, as long as you give appropriate credit to the original author(s) and the source, provide a link to the Creative Commons licence, and indicate if changes were made. The images or other third party material in this article are included in the article's Creative Commons licence, unless indicated otherwise in a credit line to the material. If material is not included in the article's Creative Commons licence and your intended use is not permitted by statutory regulation or exceeds the permitted use, you will need to obtain permission directly from the copyright holder. To view a copy of this licence, visit http://creativecommons.org/licenses/by/4.0/.

\section{References}

1. Guz' AN (1983) Mechanics of the brittle failure of materials with initial stress. Int Appl Mech 19(4):293308

2. Cottrell, A. H. (n.d.) Theory of brittle fracturee in steel and similar metals. Trans Met Soc AIME, 212

3. Jayatilaka ADS, Trustrum K (1977) Statistical approach to brittle fracture. J Mater Sci 12(7):1426-1430. https://doi.org/10.1007/BF00540858

4. Lawn BR (1993) Fracture of brittle solids, 2nd edn. Cambridge University Press, Cambridge

5. Ravi-Chandar K, Yang B (1997) On the role of microcracks in the dynamic fracture of brittle materials. J Mech PhysSolids 45(4):535-563. https://doi.org/10.1016/S0022-5096(96)00096-8

6. Wawersik WR, Fairhurst C (1970) A study of brittle rock fracture in laboratory compression experiments. Int J Rock Mech Min Sci 7(5):561-575. https://doi.org/10.1016/0148-9062(70)90007-0

7. Bocca P, Carpinteri A, Valente S (1991) Mixed mode fracture of concrete. Int J Solids Struct 27(9):11391153. https://doi.org/10.1016/0020-7683(91)90115-V

8. Malvar J, Warren G (1988) Fracture energy for three-point bend tests on single-edge notched beams. California

9. Petersson, P.-E. (1981). Crack growth and development of fracture zones in plain concrete and similar materials

10. Bleyer J, Roux-Langlois C, Molinari JF (2017) Dynamic crack propagation with a variational phase-field model: limiting speed, crack branching and velocity-toughening mechanisms. Int J Fract 204(1):79-100. https://doi.org/10.1007/s10704-016-0163-1

11. Kachanov M (1986) On crack - microcrack interactions. Int J Fract 30:65-72

12. Brencich A, Carpinteri A (1998) Stress field interaction and strain energy distribution between a stationary main crack and its process zone. Eng Fract Mech 59(6):797-814. https://doi.org/10.1016/S0013-7944(97 00158-6

13. Nagaraja Rao GM, Murthy CRL (2001) Dual role of microcracks: toughening and degradation. Can Geotech J 38(2):427-440. https://doi.org/10.1139/cgj-38-2-427

14. Kachanov M (1993) Elastic solids with many cracks and related problems. In Advances in Applied Mechanics (Vol. 30, pp. 259-445). https://doi.org/10.1192/bjp.111.479.1009-a

15. Rubinstein AA (1985) Macrocrack interaction with semi-infinite microcrack array. Int J Fract 27(2):113119. https://doi.org/10.1007/BF00040390

16. Rose LRF (1986) Microcrack interaction with a main crack. Int J Fract 31(3):233-242. https://doi. org/10.1007/BF00018929

17. Petrova V, Tamuzs V, Petrova V (2000) A survey of macro-microcrack interaction problems. Appl Mech Rev 53(5):117-146. https://doi.org/10.1115/1.3097344

18. Tamuzs V, Romalis N, Petrova V (1993) Influence of microcracks on thermal fracture of macrocrack. Theor Appl Fract Mech 19(3):207-225. https://doi.org/10.1016/0167-8442(93)90022-4

19. Milios J, Spathis G (1988) Dynamic interaction of a propagating crack with a hole boundary. Acta Mech 72(3-4):283-295. https://doi.org/10.1007/BF01178314

20. Theocaris PS, Milios J (1981) Crack-arrest at a bimaterial interface. Int J Solids Struct 17(2):217-230. https://doi.org/10.1016/0020-7683(81)90077-9

21. Theocaris PS, Milios J (1981) Crack arrest modes of a transverse crack going through a longitudinal crack or a hole. J Eng Mater Technol Transact ASME 103(2):177-182. https://doi.org/10.1115/1.3224991

22. Hu KX, Chandra A, Huang Y (1993) Multiple void-crack interaction. Int J Solids Struct 30(11):1473-1489. https://doi.org/10.1016/0020-7683(93)90072-F

23. Charalambides PG, McMeeking RM (1987) Finite element method simulation of crack propagation in a brittle microcracking solid. Mech Mater 6(1):71-87. https://doi.org/10.1016/0167-6636(87)90023-8 
24. Ortiz M, Pandolfi A (1999) Finite-deformation irreversible cohesive elements for three-dimensional crackpropagation analysis. Int J Numer Methods Eng 44(9):1267-1282. https://doi.org/10.1002/(SICI)1097-0207 (19990330)44:9<1267::AID-NME486>3.0.CO;2-7

25. Fries TP, Belytschko T (2010) The extended/generalized finite element method: an overview of the method and its applications. Int J Numer Methods Eng 84:253-304. https://doi.org/10.1002/nme

26. Belytschko T, Liu WK, Moran B, Elkhodary K (2013) Nonlinear finite elements for continua and structures. John Wiley \& sons

27. Wang H, Liu Z, Xu D, Zeng Q, Zhuang Z, Chen Z (2016) Extended finite element method analysis for shielding and amplification effect of a main crack interacted with a group of nearby parallel microcracks. Int J Damage Mech 25(1):4-25. https://doi.org/10.1177/1056789514565933

28. Budyn É, Zi G, Moës N, Belytschko T (2004) A method for multiple crack growth in brittle materials without remeshing. Int J Numer Methods Eng 61(10):1741-1770. https://doi.org/10.1002/nme.1130

29. Ren X, Guan X (2017) Three dimensional crack propagation through mesh-based explicit representation for arbitrarily shaped cracks using the extended finite element method. Eng Fract Mech 177:218-238. https://doi.org/10.1016/j.engfracmech.2017.04.007

30. Silling, S. A. (2000). Reformulation-of-elasticity-theory-for-discontinuities-and-long-rangeforces_2000_Journal-of-the-mechanics-and-physics-of-solids, 48, 175-209

31. Silling SA, Weckner O, Askari E, Bobaru F (2010) Crack nucleation in a peridynamic solid. Int J Fract 162(1-2):219-227. https://doi.org/10.1007/s10704-010-9447-z

32. Huang D, Lu G, Qiao P (2015) An improved peridynamic approach for quasi-static elastic deformation and brittle fracture analysis. Int J Mech Sci 94-95:111-122. https://doi.org/10.1016/j.ijmecsci.2015.02.018

33. Ha YD, Bobaru F (2011) Characteristics of dynamic brittle fracture captured with peridynamics. Eng Fract Mech 78(6):1156-1168. https://doi.org/10.1016/j.engfracmech.2010.11.020

34. Bobaru F, Hu W (2012) The meaning, selection, and use of the peridynamic horizon and its relation to crack branching in brittle materials. Int J Fract 176(2):215-222. https://doi.org/10.1007/s10704-012-9725-Z

35. Basoglu MF, Zerin Z, Kefal A, Oterkus E (2019) A computational model of peridynamic theory for deflecting behaviour of crack propagation with micro-cracks. Comput Mater Sci 162(November 2018): 33-46. https://doi.org/10.1016/j.commatsci.2019.02.032

36. Vazic B, Oterkus S, Wang H, Oterkus E, Diyaroglu C (2017) Dynamic propagation of a macrocrack interacting with parallel small cracks. AIMS Mater Sci 4(1):118-136. https://doi.org/10.3934 /matersci.2017.1.118

37. Yoffe EH (1951) LXXV. The moving Griffith crack. London, Edinburgh, Dublin Philos Mag J Sci 42(330): 739-750

38. Silling SA, Epton M, Weckner O, Xu J, Askari E (2007) Peridynamic states and constitutive modeling. J Elast 88:151-184. https://doi.org/10.1007/s10659-007-9125-1

39. Yang RS, Ding CX, Yang LY, Xu P, Chen C (2018) Hole defects affect the dynamic fracture behavior of nearby running cracks. Shock Vib 2018:1-8. https://doi.org/10.1155/2018/5894356

40. Silling SA, Askari E (2005) A meshfree method based on the peridynamic model of solid mechanics. Comput Struct 83(17-18):1526-1535. https://doi.org/10.1016/j.compstruc.2004.11.026

41. Kilic B, Madenci E (2010) An adaptive dynamic relaxation method for quasi-static simulations using the peridynamic theory. Theor Appl Fract Mech 53(3):194-204. https://doi.org/10.1016/j.tafmec.2010.08.001

42. Madenci E, Oterkus E (2014) Peridynamic theory and its applications. Springer Science+Business Media, New York

43. Silling SA, Bobaru F (2005) Peridynamic modeling of membranes and fibers. Int J Non-Linear Mech 40(23):395-409. https://doi.org/10.1016/j.ijnonlinmec.2004.08.004

44. Chen YZ, Chen RS (1997) Interaction between curved crack and elastic inclusion in an infinite plate. Appl Mech 67:566-575. https://doi.org/10.1007/BF01175594

45. Gong SX, Horii H (1989) General solution to the problem of microcracks near the tip of a main crack. J Mech Phys Solids 37:27-46. https://doi.org/10.1016/0022-5096(87)90003-2

46. Romalis NB, Tamuzh VP (1983) Propagation of a main crack in a body with distributed microcracks. In Fifth All-Union Conference on the Mechanics of Polymer (pp. 20-23)

47. Madenci E, Oterkus S (2016) Ordinary state-based peridynamics for plastic deformation according to von Mises yield criteria with isotropic hardening. J Mech PhysSolids 86:192-219. https://doi.org/10.1016/j. jmps.2015.09.016

48. Döll W (1975) Investigations of the crack branching energy. Int J Fract 11(1):184-186

49. Chandar KR, Knauss WG (1982) Dynamic crack-tip stresses under stress wave loading -A comparison of theory and experiment. Int J Fract 20(3):209-222. https://doi.org/10.1007/BF01140336 